\title{
New Geophysical Technique for Mineral Exploration and Mineral Discrimination Based on Electromagnetic Methods
}

DE-FC26-04NT42081

ANNUAL PROGRESS REPORT

Principal Author: Michael S. Zhdanov

University of Utah, Salt Lake City, UT 84112

Reporting Period Start Date: April 9, 2004

Reporting Period End Date: April 92005

July 2005 


\begin{abstract}
The research during the first year of the project was focused on developing the foundations of a new geophysical technique for mineral exploration and mineral discrimination, based on electromagnetic (EM) methods. The proposed new technique is based on examining the spectral induced polarization effects in electromagnetic data using modern distributed acquisition systems and advanced methods of 3-D inversion.

The analysis of IP phenomena is usually based on models with frequency dependent complex conductivity distribution. One of the most popular is the Cole-Cole relaxation model. In this progress report we have constructed and analyzed a different physical and mathematical model of the IP effect based on the effective-medium theory. We have developed a rigorous mathematical model of multi-phase conductive media, which can provide a quantitative tool for evaluation of the type of mineralization, using the conductivity relaxation model parameters. The parameters of the new conductivity relaxation model can be used for discrimination of the different types of rock formations, which is an important goal in mineral exploration. The solution of this problem requires development of an effective numerical method for EM forward modeling in 3-D inhomogeneous media. During the first year of the project we have developed a prototype 3-D IP modeling algorithm using the integral equation (IP) method. Our IE forward modeling code INTEM3DIP is based on the contraction IE method, which improves the convergence rate of the iterative solvers. This code can handle various types of sources and receivers to compute the effect of a complex resistivity model. We have tested the working version of the INTEM3DIP code for computer simulation of the IP data for several models including a southwest US porphyry model and a Kambalda-style nickel sulfide deposit. The numerical modeling study clearly demonstrates how the various complex resistivity models manifest differently in the observed EM data. These modeling studies lay a background for future development of the IP inversion method, directed at determining the electrical conductivity and the intrinsic chargeability distributions, as well as the other parameters of the relaxation model simultaneously. The new technology envisioned in this proposal, will be used for the discrimination of different rocks, and in this way will provide an ability to distinguish between uneconomic mineral deposits and the location of zones of economic mineralization and geothermal resources.
\end{abstract}




\section{DISCLAIMER:}

"This report was prepared as an account of work sponsored by an agency of the United States Government. Neither the United States Government nor any agency thereof, nor any of their employees, makes any warranty, express or implied, or assumes any legal liability or responsibility for the accuracy, completeness, or usefulness of any information, apparatus, product, or process disclosed, or represents that its use would not infringe privately owned rights. Reference herein to any specific commercial product, process, or service by trade name, trademark, manufacturer, or otherwise does not necessarily constitute or imply its endorsement, recommendation, or favoring by the United States Government or any agency thereof. The views and opinions of authors expressed herein do not necessarily state or reflect those of the United States Government or any agency thereof." 


\section{Contents}

1 INTRODUCTION: PROJECT RATIONALE AND STRATEGY

2 EXECUTIVE SUMMARY 15

$\begin{array}{lll}3 & \text { THEORY } & 17\end{array}$

3.1 Basic characteristic of IP methods . . . . . . . . . . . . . . . 17

3.2 Construction and analysis of the conductivity relaxation model . 22

3.2.1 Cole-Cole conductivity relaxation models . . . . . . . . . 22

3.2.2 Mathematical modeling of the electrical conductivity of a composite medium . . . . . . . . . . . . 24

3.2 .3 Asymptotics of the effective conductivity . . . . . . . 28

4 EXPERIMENTAL 33

4.1 Development of the 3-D EM-IP forward modeling system . . . . 33

4.2 Application of INTEM3DIP to the southwest U.S. porphyry model 33 4.2 .1 1-D Model . . . . . . . . . . . . . . . . . . . . . 34

$4.2 .2 \quad 3$-D models . . . . . . . . . . . . . . . . . . . 37

4.3 Application of INTEM3DIP to the model of a Kambalda-style nickel sulfide deposit . . . . . . . . . . . . . . . . 48

4.3.1 Trench-Williams Model 1 (TWM1) . . . . . . . . . . . 50

4.3.2 Trench-Williams Model 2 (TWM2) . . . . . . . . . . . 50

4.3.3 Trench-Williams Model 3 (TWM3) . . . . . . . . . . . . 51

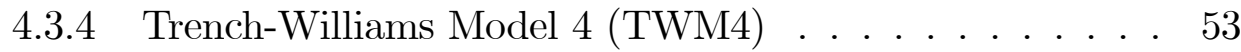

5 RESULTS AND DISCUSSION 57

6 CONCLUSIONS $\quad 59$

7 REFERENCES $\quad 61$

$\begin{array}{ll}\text { CONTENTS } & 5\end{array}$ 


\section{List of Figures}

3-1 Sketch of the potential waveform for a current injected into nonpolarizable and polarizable ground. Panel (a) shows the case of non-polarizable ground and (b) shows that of polarizable ground. 18

3-2 Representation of IP effects in the time domain. Panel (a) shows the injected current versus time. The chargeability can be represented as (b), the ratio of potential decay, or (c), the area under the decay curve, to the maximum potential. . . . . . . . . . . 19

3-3 Representation of IP effects in the frequency domain. Panel (a) shows percent frequency effect, and (b) the phase difference between the current and the potential. . . . . . . . . . . . . 21

3-4 Pseudosection representation. The data are plotted at the midpoint of a transmitter and receiver pair versus a separation in-

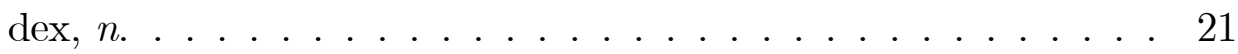

3-5 Examples of typical complex resistivity curves with the ColeCole model parameters. The upper panel shows the real part of the complex resistivity, while the bottom panel presents the imaginary part of the complex resistivity. . . . . . . . . . 23

3-6 A model of a two-phase composite conductive medium . . . . . 25

3-7 The real part of the effective conductivity. The conductivity contrast, $p$, is the code of the curves. . . . . . . . . . . . 29

3-8 The imaginary part of the effective conductivity. The conductivity contrast, $p$, is the code of the curves. . . . . . . . . . 30

4-1 A resistivity/ip model of a "typical" porphyry copper system in the southwestern U.S. . . . . . . . . . . . . . . . . . 34

4-2 1-D model obtained from the conceptual model of a "typical" southwest U.S. porphyry deposit. . . . . . . . . . . . . . . 35

4-3 Apparent resistivity pseudosection obtained by the INTEM3DIP code for a 1-D IP model. . . . . . . . . . . . . . . . . . . 36

4-4 Pseudosection of the phase of apparent resistivity obtained by the INTEM3DIP code for a 1-D IP model. . . . . . . . . . . . . 36

4-5 One of the 3-D models established from the conceptual model for a southwest U.S. porphyry deposit. . . . . . . . . . . . . . 37

4-6 Apparent resistivity obtained by the INTEM3DIP code for 3-D Model 1. . . . . . . . . . . . . . . . . . 38 
4-7 The phase of apparent resistivity obtained by the INTEM3DIP code for 3-D Model 1. . . . . . . . . . . . . . . . . . . 38

4-8 Apparent resistivity obtained by the INTEM3DIP code for 3-D Model 1. In this modeling, the imaginary part of the conductivities is set to zero to eliminate the IP effects. . . . . . . . . . . 38

4-9 The phase of apparent resistivity obtained by the INTEM3DIP code for 3-D Model 1. In this modeling, the imaginary part of the conductivities is set to zero to eliminate the IP effects. . . . 38

4-10 Vertical section of 3-D Model 2 of southwest U.S. porphyry deposit. 39

4-11 Apparent resistivity pseudosection obtained by INTEM3DIP for 3-D Model 2. . . . . . . . . . . . . . . . . . . 40

4-12 The phase pseudosection of apparent resistivity obtained by INTEM3DIP for 3-D Model 2. . . . . . . . . . . . . . . . . .

4-13 Plot of the Ex component of the observed electric field normalized by the background electric field Ex for 3-D Model 2. Each curve corresponds to one source location. . . . . . . . . . . . . 41

4-14 Vertical section of 3-D Model 3 of southwest U.S. porphyry deposit. 41 4-15 Apparent resistivity pseudosection obtained by INTEM3DIP for 3-D Model 3. . . . . . . . . . . . . . . . . . . . . 42

4-16 The phase pseudosection of apparent resistivity obtained by INTEM3DIP for 3-D Model 3. . . . . . . . . . . . . . . . . . . 42

4-17 Plot of the Ex component of the observed electric field normalized by the background electric field Ex for 3D Model 3. Each curve corresponds to one source location. . . . . . . . . . . . . 43

4-18 Vertical section of 3-D Model 4 of southwest U.S. porphyry deposit. 43

4-19 Apparent resistivity pseudosection obtained by INTEM3DIP for 3-D Model 4. . . . . . . . . . . . . . . . . . . . . . . . 44

4-20 The phase pseudosection of apparent resistivity obtained by INTEM3DIP for 3-D Model 4. . . . . . . . . . . . . . . . . . 44

4-21 Plot of the Ex component of the observed electric field normalized by the background electric field Ex for 3-D Model 4. Each curve corresponds to one source location. . . . . . . . . . . . . 45

4-22 Vertical section of 3-D Model 5 of southwest U.S. porphyry deposit. 45

4-23 Apparent resistivity pseudosection obtained by INTEM3DIP for 3-D Model 5. . . . . . . . . . . . . . . . . 46

4-24 The phase pseudosection of apparent resistivity obtained by INTEM3DIP for 3-D Model 5. . . . . . . . . . . . . . . . .

4-25 Plot of the Ex component of the observed electric field normalized by the background electric field Ex for 3-D Model 5. Each curve corresponds to one source location. . . . . . . . . . . .

4-26 Cartoon of the cross-section of the Kambalda NiS deposit after Trench and Williams (1994). . . . . . . . . . . . . .

4-27 Cross-section of the total resistivity along the $y=0 \mathrm{~m}$ profile for the Kambalda-style NiS orebody used for forward modeling the FEM response. . . . . . . . . . . . . . . . . . . 
4-28 A 3-D view of Trench-Williams Model 1 (TWM1). . . . . . . . . 51

4-29 Pseudosections of apparent resistivity and phase for TrenchWilliams Model 1. . . . . . . . . . . . . . . . . . . 52

4-30 A 3-D view of Trench-Williams Model 2 (TWM2). . . . . . . . . 53

4-31 Pseudosections of apparent resistivity and phase for TrenchWilliams Model 2. . . . . . . . . . . . . . . . 54

4-32 A 3-D view of Trench-Williams Model 3 (TWM3). . . . . . . . . 55

4-33 Pseudosections of apparent resistivity and phase for TrenchWilliams Model 3. . . . . . . . . . . . . . . . . . . . 55

4-34 A 3-D view of Trench-Williams Model 4 (TWM4). . . . . . . . 56

4-35 Pseudosections of apparent resistivity and phase for TrenchWilliams Model 4. . . . . . . . . . . . . . . . . . 56 


\section{INTRODUCTION: PROJECT RATIO- NALE AND STRATEGY}

One of the major problems of mineral exploration is the ability to reliably distinguish between uneconomic mineral deposits and economic mineralization. While the mining industry uses many geophysical methods to locate mineral deposits, until recently, there was no reliable technology for mineral resources identification and characterization. The main goal of this project is to develop a new geophysical technique for subsurface material characterization, mineral exploration and mineral discrimination, based on electromagnetic (EM) methods. The proposed new technique will be based on examining the spectral induced polarization (IP) effects in EM data using the modern distributed acquisition systems and advanced methods of $3-\mathrm{D}$ modeling and inversion.

This project is undertaken using multipartner collaboration between the University of Utah, four major mining companies, Kennecott Exploration Company located in Utah, USA; BHP Billiton World Exploration Inc. with offices in the USA, Canada, Australia; Placer Dome Inc. with offices in Canada and the USA, Phelps Dodge Mining Company with the offices in USA and overseas, and Zonge Engineering and Research Organization, Inc. located in Tucson, Arizona, an equipment manufacturer and service provider to the mining industry.

The Center for Electromagnetic Modeling and Inversion (CEMI) at the University of Utah has long-term experience in developing forward and inverse solutions for EM methods of geophysics, and in providing interpretation insight through model studies. The research results developed by CEMI have contributed significantly in improving the effectiveness of EM techniques in mining, petroleum, geothermal, and engineering applications. Further research will be supported by the Center for High Performance Computing (CHPC) at the University of Utah, which is responsible for providing high-end computer services to advanced programs in computational sciences and simulations and has core competencies in operating largescale computing resources, advanced networking and scientific computing, and simulations.

Kennecott Exploration Company and its affiliate companies are members of the Rio Tinto group of companies, which is a leader in the international mining industry, helping to meet the growing national and global needs for minerals and metals. BHP Billiton was formed from a merger between two highly complementary companies - BHP and Billiton. It is one of the world's premier mining companies, with a portfolio of best-in-class mining and metals operations. Placer Dome is the world's fifth largest gold mining company. Phelps Dodge Mining Company is one of the world's leading producers of copper and molybdenum. Zonge Engineering and Research Organization, Inc. is 
one of the major mining equipment manufacturing and service providing companies. Over the years, Zonge has developed a complete line of transmitters, receivers, and peripheral equipment used for all types of electrical geophysical surveys, including time and frequency domain IP, controlled source magnetotelluric (CSAMT), transient or pulse EM (TEM), frequency domain EM, and natural source MT and audio MT systems.

The multi-partner collaboration between the academic institution, the University of Utah, and the mining industry will help to identify appropriate mining targets and conduct field experiments for practical demonstration of the developed new technology, which will be an important part of the proposed research project.

In summary, the main goal of this project is to develop new principles and methods that will result in a new enabling geophysical technology for mineral exploration and mineral discrimination based on EM data. This new technology will address the following problems:

1) to develop a new geophysical technique for deep sensing of rocks, minerals, and geological structures, based on the analysis of 3-D distribution of complex conductivity inferred from the observed EM geophysical data;

2) to increase the resolution, stability, and reliability of 3-D EM and IP data inversion by applying regularization methods based on focusing stabilizers;

3) to speed up computations and to enable solution of largescale 3-D EM forward and inverse problems using parallel computing;

4) to improve the definition of targets and subsurface material characterization, and to increase the depth of investigation, as required by the research priorities identified in the Exploration and Mining Technology Roadmap (September 2002);

$5)$ to enable enhanced and more efficient resource identification and characterization by geophysical exploration methods, with less impact on the environment.

The IP effect is caused by the complex EM phenomena that accompany current flow in the earth. These phenomena take place in a heterogeneous medium representing rock formations in the areas of mineralization. It was demonstrated more than twenty years ago in the pioneer papers by Zonge and Wynn, 1975, and Pelton et al., 1978, that the IP effect may be used to separate the responses of economic polarized targets from other anomalies. However, until recently this idea has had very limited practical application because of the difficulties in recovering the induced polarization parameters from the observed electromagnetic (EM) data, especially in the case of 3-D interpretation required for efficient exploration of the mining targets, and because of the absence of adequate composite conductivity models of the rock formations.

The quantitative interpretation of IP data in a complex 3-D environment is a very challenging problem. The analysis of IP phenomena is usually based on models with frequency dependent complex conductivity distribution. One of the most popular is the Cole-Cole relaxation model and its different modifications. The parameters of the conductivity relaxation model can be used for 
discrimination of the different types of rock formations, which is an important goal in mineral exploration. Until recently, these parameters have been determined mostly in the physical lab by direct analysis of the rock samples. In this project we develop and investigate a new geophysical technique for determining 3-D distribution of the same parameters of the rock formations in the field from the geophysical observations. In addition, we introduce a new composite geoelectrical model of the rock formations, which generates a conductivity relaxation model with the parameters directly related by analytical expressions to the physical characteristics of the microstructure of the rocks and minerals (microgeometry and conductivity parameters). A new composite geoelectrical model provides more realistic representation of the complex rock formations than the conventional unimodal conductivity models. It will allow us to model the relationships between the physical characteristics of different types of minerals (e.g. conductivities and sulfide grain sizes) and the parameters of the relaxation model. As a result, the new method will allow us to determine the internal structure and physical characteristics of the different rock formations by remote geophysical techniques without drilling the wells.

The goal of the EM geophysical survey will be to determine the electrical conductivity and the intrinsic chargeability distributions as well as the other parameters of the relaxation model simultaneously. The recovered parameters of the relaxation model will be used for the discrimination of different rocks, and in this way will provide the ability to distinguish between uneconomic mineral deposits and zones of economic mineralization using geophysical remote sensing technology, which is one of the research priorities identified in the Exploration and Mining Technology Roadmap (September 2002, Exhibit 1: Remote Sensing Technology).

The solution of this problem requires development of effective numerical methods for EM forward modeling and inversion in inhomogeneous media. These problems are extremely difficult, especially in three-dimensional case. The difficulties arise even in the forward modeling because of the huge size of the numerical problem to be solved to adequately represent the complex 3-D distribution of EM parameters of the media, required in mining exploration. As the result, the computer simulation time and the memory requirements could be excessive even for the practically realistic models.

During the first research year of the project, our efforts were focused on constructing a new conductivity relaxation model and on developing an effective numerical method for fast and accurate modeling the IP effect for different complex 3-D geoelectrical structures. This annual report contains a description of basic principles for mathematical modeling of electrical conductivity of the composite medium and the preliminary results of our numerical modeling study. 


\section{EXECUTIVE SUMMARY}

The research during the first year of the project was focused on developing the foundations of a new geophysical technique for mineral exploration and mineral discrimination, based on electromagnetic methods. The proposed new technique is based on examining the spectral induced polarization effects in electromagnetic data using modern distributed acquisition systems and advanced methods of $3-\mathrm{D}$ inversion.

The induced polarization (IP) effect is caused by the complex electrochemical reactions that accompany current flow in the earth. The analysis of IP phenomena is usually based on models with frequency dependent complex conductivity distribution. One of the most popular is the Cole-Cole relaxation model. In this progress report we have constructed and analyzed a different physical and mathematical model of the IP effect based on the effective-medium theory. A new composite geoelectrical model provides more realistic representation of complex heterogeneous rock formations than conventional unimodal conductivity models. It allows us to model the relationships between the physical characteristics of different types of minerals (e.g. conductivities and sulfide grain sizes) and the parameters of the conductivity relaxation model. This new conductivity relaxation model can be used for discrimination of different types of rock formations, which is an important goal in mineral exploration.

The solution of this problem requires development of effective numerical methods for EM forward modeling in 3-D inhomogeneous media. During the first year of the project we have developed a prototype 3-D IP modeling algorithm using the integral equation (IE) method. Our IE forward modeling code INTEM3DIP is based on the contraction IE method, which improves the convergence rate of the iterative solvers. This code can handle various types of sources and receivers to compute the effect of a complex resistivity model. We have tested the working version of the INTEM3DIP code for computer simulation of the IP data for several models of typical mineral deposits.

We have studied a resistivity/IP model of a typical porphyry copper system in the southwestern U. S. This model is characterized by potentially strong EM coupling as well as IP effects. We considered a simplified 1-D model of the southwest U.S. porphyry deposit, and several more complex 3-D models.

We have also simulated an EM-IP survey above a Kambalda-style, nickel sulfide deposit located beneath a complicated regolith horizon. In the general exploration model, the regolith is highly conductive and overlays the resistive host rock and conductive target. To the explorer trying to use EM methods, this inhomogeneous regolith creates "false" anomalies due to conductive sedimentary and weathered units, and the extensive blanket of salt lake sediments in certain areas effectively masks the EM response from the target. All these factors point towards the associated analysis of EM-IP responses being an ex- 
tremely challenging problem, which is confirmed by our computer simulation.

The numerical modeling study clearly demonstrates how the various complex resistivity models manifest differently in the observed electromagnetic data. This modeling study lays a background for future development of the IP inversion method, directed at determining the electrical conductivity and the intrinsic chargeability distributions, as well as the other parameters of the relaxation model simultaneously. The new technology envisioned in this project, will be used for the discrimination of different rocks, and in this way will provide an ability to distinguish between uneconomic mineral deposits and the location of zones of economic mineralization and geothermal resources. 


\section{THEORY}

\subsection{Basic characteristic of IP methods}

The development of the IP method can be traced back to the 1950s, when both mining and petroleum companies were actively looking into the application of this method for mineral exploration. The physical-mathematical principles of the IP effect were originally formulated in pioneered works by Wait (1959) and Sheinman (1969). However, this method did not find wide application in US industry until after the work of Zonge and his associates at the Zonge Engineering and Research Organization (Zonge and Wynn, 1975) and Pelton and Ward at the University of Utah (1978). Significant contribution to the development of the IP method was made, also, by Professor Wait $(1959,1982)$, and by the research team at Kennecott in 1965-1977 (Nelson, 1997).

The IP phenomenon is usually associated with charging and discharging effects in the ground in the process of current injecting. Figure 3-1 schematically illustrates the typical IP effect. Suppose that we inject current into the ground and measure the electric potential difference between two electrodes. In the absence of the IP effect, if the ground is not chargeable (not polarizable), the measured potential will vary with time exactly with the same waveform as the electric current in the source, without any delay in rise-up or fall-down of the potential. However, if the ground is chargeable (polarizable), the potential will rise up and fall down with some time delay. This phenomenon is usually explained as membrane polarization which occurs when pore space narrows to within several boundary layers of thickness, or as electrode polarization, which occurs when pore space is blocked by metallic particles. We will demonstrate below, however, that the same phenomenon can be mathematically explained by the propagation of a current in the complex multi-phase composite geoelectrical model. The goal of the conventional IP survey is to detect these phenomena for imaging the subsurface structures by measuring the chargeability of the rock formations.

In practice, two types of IP data can be acquired in exploration: time domain and frequency domain data. Figures 3-2b and 3-2c present typical time domain potential curves obtained by IP measurement. When the current is injected (turn-on signal in the source) into the ground (3-2a), the potential rises up immediately, but it takes some time to reach the maximum. The same behavior of the potential is observed when the current is terminated (turn-off signal in the source): the potential does not fall down to zero immediately, but it takes some time to decay to zero. The time domain chargeability is defined as the ratio of the potential at some time after turn-off, $\phi_{s}(t)$, to the maximum 

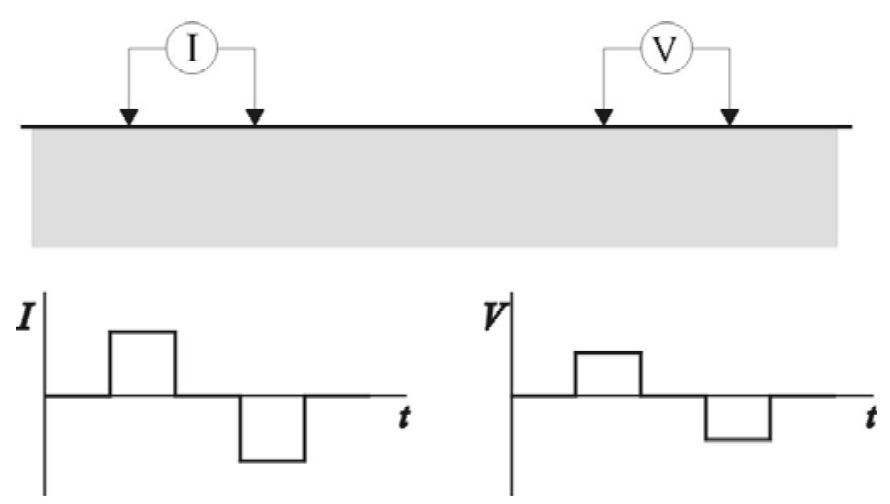

(a)
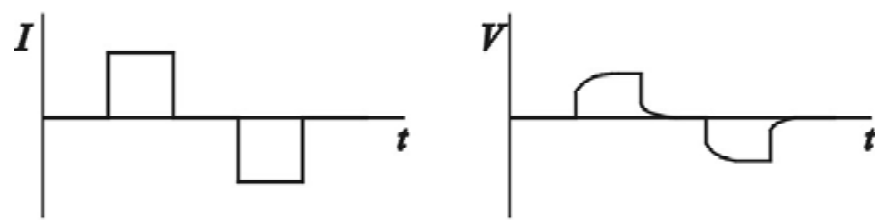

(b)

Figure 3-1 Sketch of the potential waveform for a current injected into nonpolarizable and polarizable ground. Panel (a) shows the case of non-polarizable ground and (b) shows that of polarizable ground.

value of the potential, $\phi_{\eta}(3-2)$ :

$$
M(t)=\phi_{s}(t) / \phi_{\eta},
$$

or as the ratio of the integral of the potential decay curve after turn-off to the maximum potential:

$$
M=\frac{1}{\phi_{\eta}} \int_{t_{1}}^{t_{2}} \phi_{s}(t) d t .
$$

In the frequency domain, the IP effect can be represented as a percent frequency effect $(\mathrm{PFE})$, which is defined as the relative difference between the apparent resistivity with a higher frequency, $\rho_{a}^{f_{1}}$, from that with a lower frequency, $\rho_{a}^{f_{2}}$, normalized by the apparent resistivity with the high frequency, in percents (Figure 3-3a):

$$
P F E=\left(\frac{\rho_{a}^{f_{1}}-\rho_{a}^{f_{2}}}{\rho_{a}^{f_{1}}}\right) \times 100 .
$$

Another representation of IP effect in the frequency domain is the phase difference between the current in the source and the measured potential depending on frequencies of the source (Figure 3-3 b).

Note that the conventional IP method in the frequency domain is very similar to the DC resistivity method in that both methods use the electric field 


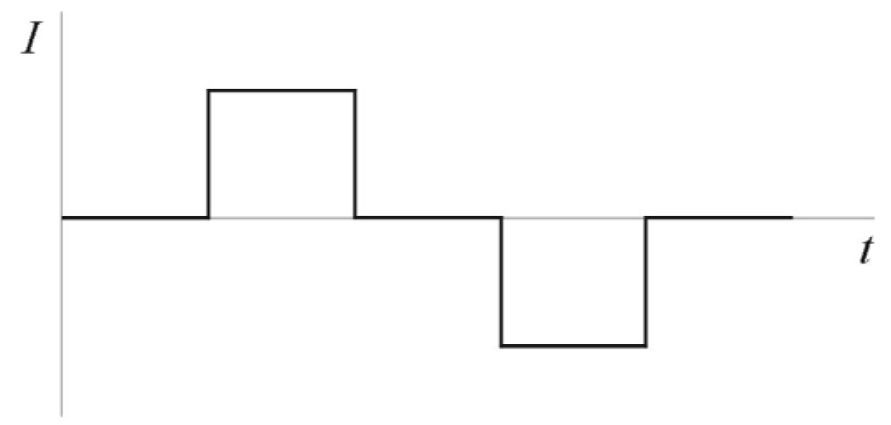

(a)

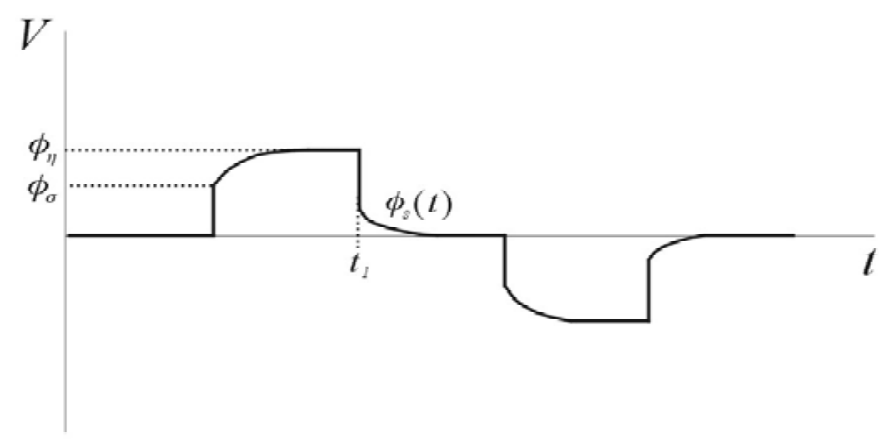

(b)

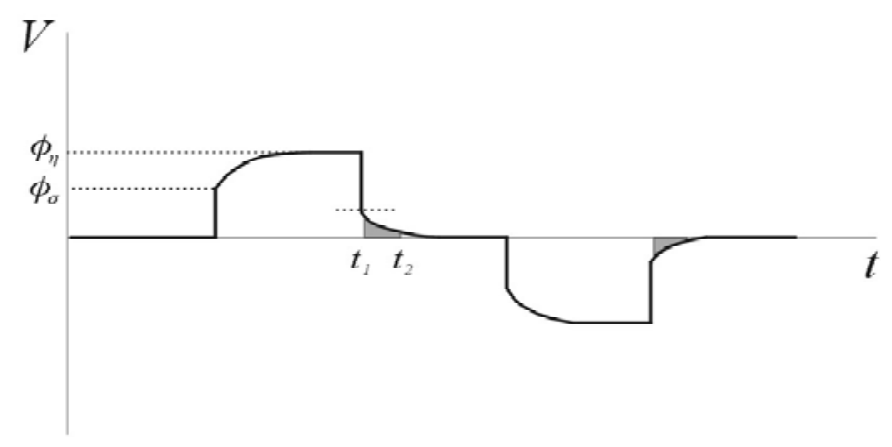

(c)

Figure 3-2 Representation of IP effects in the time domain. Panel (a) shows the injected current versus time. The chargeability can be represented as (b), the ratio of potential decay, or (c), the area under the decay curve, to the maximum potential. 
at a very low frequency, and IP data acquisition systems and interpretation techniques are very similar to those of the DC resistivity methods. Therefore one can define the apparent resistivity according to the standard DC resistivity formula:

$$
\rho_{a}(\omega)=K \frac{\Delta U(\omega)}{I(\omega)},
$$

where $K$ is a geometric factor depending on the source-receiver electrodes configuration (Luo and Zhang, 1998), $\Delta U(\omega)$ is the potential measured in the receivers, and $I(\omega)$ is the strength of the injected current. For example, if we use a dipole-dipole array,

$$
K=\frac{n(n+1)(n+2)(n+3)}{2} \pi l
$$

where $l$ is the dipole length, and $n$ is the separation index between source and receiver pair (the distance between the source electrodes and receiver electrodes is equal to $n l$ ).

Note that, in the presence of the IP effect the measured potential in the frequency domain is complex; therefore, the apparent resistivity is characterized by a complex number, as well:

$$
\rho_{a}(\omega)=\operatorname{Re} \rho_{a}(\omega)+i \operatorname{Im} \rho_{a}(\omega) .
$$

The magnitude of the complex apparent resistivity is the same as the DC apparent resistivity:

$$
\rho_{a}^{D C}(\omega)=\left|\rho_{a}(\omega)\right|=\sqrt{\left(\operatorname{Re} \rho_{a}(\omega)\right)^{2}+\left(\operatorname{Im} \rho_{a}(\omega)\right)^{2}}
$$

and the phase is described as:

$$
\phi_{a}(\omega)=\tan ^{-1} \frac{\operatorname{Im} \rho_{a}(\omega)}{\operatorname{Re} \rho_{a}(\omega)}
$$

Figure 3-4 shows the data representation in the form of a pseudosection, which is a well known data representation method in resistivity or IP surveys. The data are plotted at the midpoint of a transmitter and receiver pair versus a separation index, $n$. The separation index $n$ of transmitter and receiver is defined as a distance between two adjacent stations, divided by the electric dipole length, as shown in Figure 3-4.

The apparent resistivity magnitude and phase pseudosections provide useful images of the observed data, which can be used for qualitative interpretation. We will give some examples of these pseudosections for the typical mining targets below in the section on numerical forward modeling. 


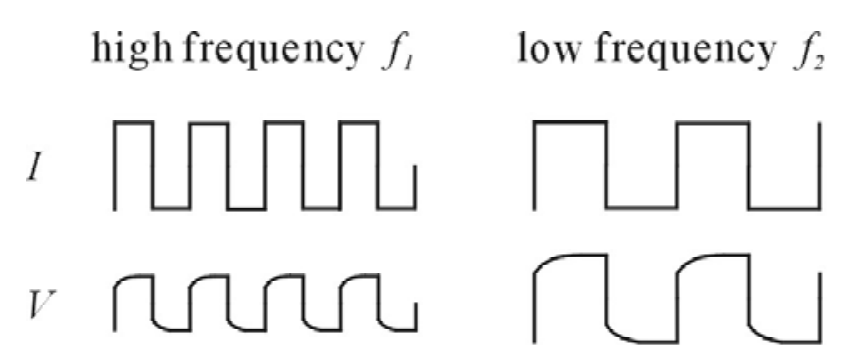

(a)

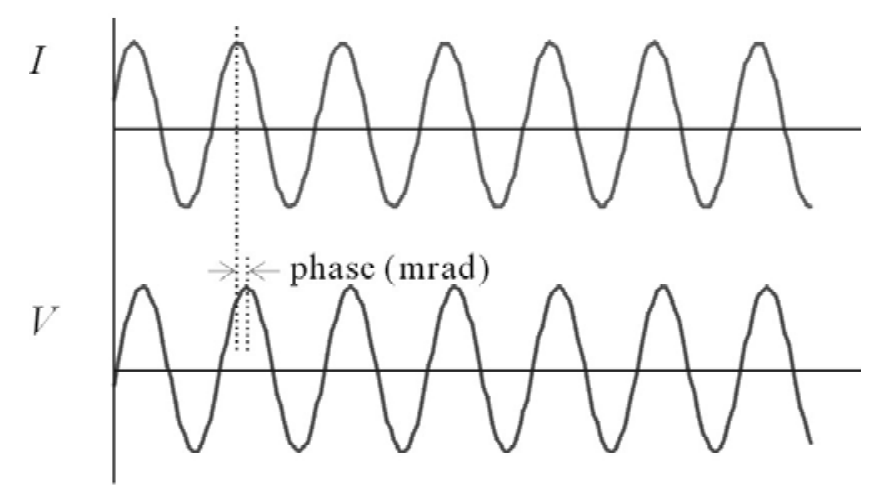

(b)

Figure 3-3 Representation of IP effects in the frequency domain. Panel (a) shows percent frequency effect, and (b) the phase difference between the current and the potential.

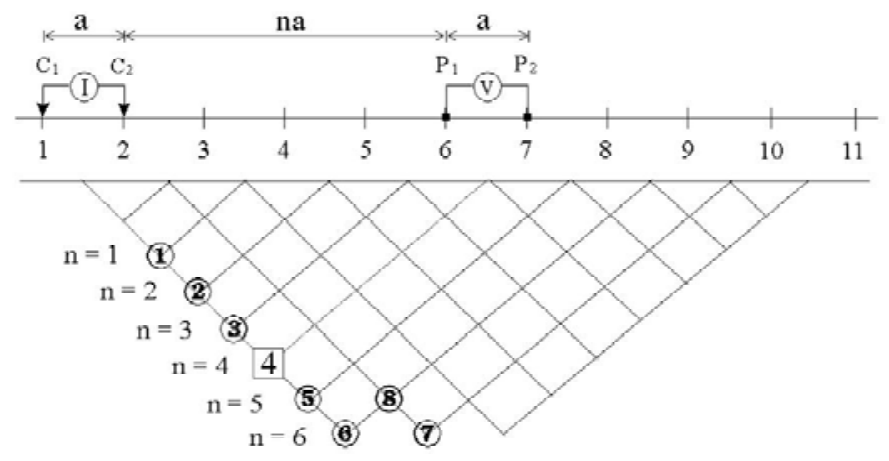

Figure 3-4 Pseudosection representation. The data are plotted at the midpoint of a transmitter and receiver pair versus a separation index, $n$. 


\subsection{Construction and analysis of the conductiv- ity relaxation model}

\subsubsection{Cole-Cole conductivity relaxation models}

It is well understood now that the effective conductivity of rocks is not necessarily a constant and real number but may vary with frequency and be complex (Shuev, R.T., and Johnson, M., 1973). There are several explanations for these properties of the effective conductivity. Most often they are explained by the physical-chemical polarization effects of the mineralized particles of the rock material, and/or by the electrokinetic effects in the poroses of the reservoir (Wait, 1959; Marshall and Madden, 1959; Luo and Zhang, 1998).

It was demonstrated in the pioneer work of Pelton (1977), that the ColeCole relaxation model (Cole and Cole, 1941) can represent well the typical complex conductivity of polarized rock formations. In the framework of this model, the complex resistivity, $\rho(\omega)$, is described by the following well known expression:

$$
\rho(\omega)=\rho\left(1-\eta\left(1-\frac{1}{1+(i \omega \tau)^{\mathrm{C}}}\right)\right),
$$

where $\rho$ is the DC resistivity [Ohm-m]; $\omega$ is the angular frequency $[\mathrm{rad} / \mathrm{sec}]$; $\tau$ is the time parameter; $\eta$ is the intrinsic chargeability (Seigel, 1959); and $C$ is the relaxation parameter. The dimensionless intrinsic chargeability, $\eta$, characterizes the intensity of the IP effect.

Figure 3-5 presents examples of the typical complex resistivity curves with the Cole-Cole model parameters defined according to the following table:

Table 1

$$
\begin{array}{rlrl} 
& \text { Model 1 } & \multicolumn{1}{c}{\text { Model 2 }} \\
\rho_{1}=2 \text { Ohm-m, } & \rho_{2}=2 \text { Ohm-m, } \\
\eta_{1}=0.5, & \eta_{2}=0.5 \\
\tau_{1}=0.4, & \tau_{2}=0.04 \\
C_{1}=0.8, & C_{2}=0.3 .
\end{array}
$$

One can see a significant difference between the solid and dashed curves in this plot, which corresponds to the different Cole-Cole models with different parameters.

Note also that the Cole-Cole curve gives us just one possible example of the relaxation model. There are several other models discussed in the geophysical 

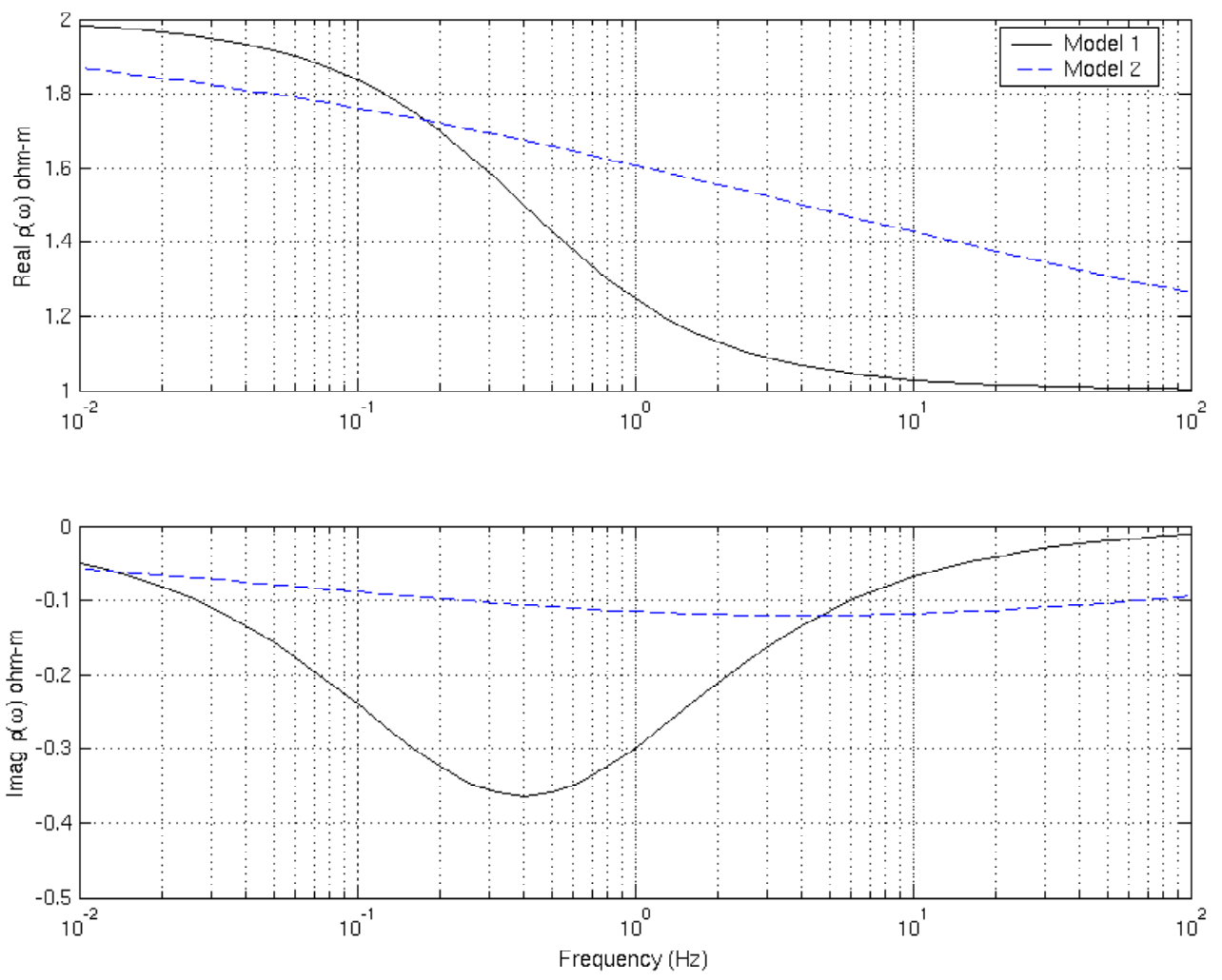

Figure 3-5 Examples of typical complex resistivity curves with the Cole-Cole model parameters. The upper panel shows the real part of the complex resistivity, while the bottom panel presents the imaginary part of the complex resistivity. 
literature (see, for example, Kamenetsky, 1997). One of the important practical questions is the relationship between the Cole-Cole model parameters and the petrophysical characteristics of mineralized rocks. There were several publications specifically dedicated to the solution of this problem. However, most of the published results provide just a qualitative indication of the correlation between Cole-Cole parameters and specific mineralization characteristics of the rocks, such as mineral grain sizes and physical properties.

\subsubsection{Mathematical modeling of the electrical conductivity of a composite medium}

One of the reasons of electrical conductivity relaxation in rocks is the heterogeneity of the formations containing microscopic inclusions of the different minerals. In the pioneered work by Wait (1982, p.77), a simplified model of the composite medium as a loading of spherical conducting particles in a resistive background was introduced. The effective conductivity for this model was determined based on the equations of the static electric field. This model provided a foundation for the phenomenological theory of induced electrical polarization.

The advances of the physical effective-medium theories (e. g., Norris et al., 1985; Kolundzija and Djordjevic, 2002) make it possible to develop a rigorous mathematical model of multi-phases heterogeneous conductive media excited by a transient EM field. The analysis of this model can provide a quantitative tool for evaluation of the type of mineralization, using the conductivity relaxation model parameters. In this section we will discuss a new approach to the solution of this problem for a model of two-phases composite media.

Let us consider a composite model formed by a homogeneous background with a conductivity $\sigma_{1}$ filled with the conductive balls of a radius $R$ with a conductivity $\sigma_{2}$ (Figure 3-6). The balls are distributed homogeneously in the lower half-space $(z \geq 0)$ at a distance between the nearby ball centers equal to $L>2 R$. The centers of the balls are located along the horizontal planes, $z=L / 2,3 L / 2, \ldots \ldots .,(3 p+1) L / 2, \ldots \ldots$ The medium is excited by the vertically propagated plane EM wave:

$$
\mathbf{E}^{b}(\mathbf{r})=\mathbf{E}^{b}(z)=\mathbf{b} e^{i k_{1} z} ; \quad E_{x, y}^{b}=b_{x, y} e^{i k_{1} z}
$$

where $k_{1}^{2}=i \omega \mu_{0} \sigma_{1}, \mathbf{b}=\left(b_{x}, b_{y}, 0\right)$ is a vector of the plane wave components at $z=0$, and $\mu_{0}$ is the free space magnetic permeability.

We can introduce anomalous electric field $\mathbf{E}^{a}$ generated by the currents induced in the balls, computed by the formula:

$$
\mathbf{E}^{a}\left(\mathbf{r}^{\prime}, \omega\right)=\iiint_{D} \widehat{\mathbf{G}}_{E}\left(\mathbf{r}^{\prime} \mid \mathbf{r}, \omega\right) \cdot\left\{\Delta \sigma\left[\mathbf{E}^{b}(\mathbf{r}, \omega)+\mathbf{E}^{a}(\mathbf{r}, \omega)\right]\right\} d v
$$

where $\Delta \sigma=\sigma_{2}-\sigma_{1}$, and $D$ is the volume occupied by all balls. 


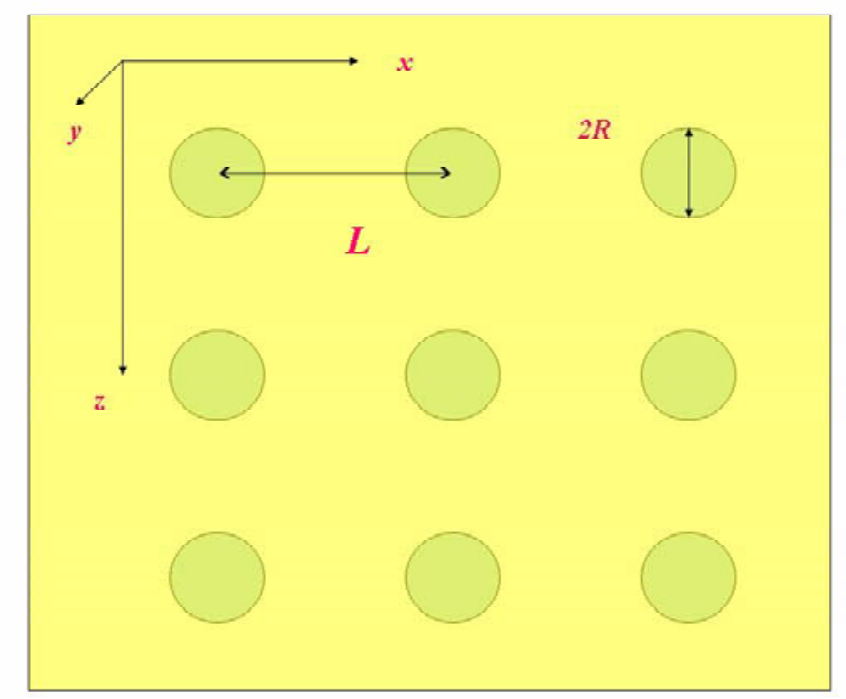

Figure 3-6 A model of a two-phase composite conductive medium

Assuming that the radius of every ball is much smaller than the wavelength in the background medium, $R \ll \lambda_{1}=2 \pi \sqrt{2} / \sqrt{\omega \mu \sigma_{1}}$, we can use the Born approximation for the anomalous field (Zhdanov, 2002):

$$
\mathbf{E}^{a}\left(\mathbf{r}^{\prime}, \omega\right)=\iiint_{D} \widehat{\mathbf{G}}_{E}\left(\mathbf{r}^{\prime} \mid \mathbf{r}, \omega\right) \cdot \Delta \sigma \mathbf{E}^{b}(z, \omega) d v
$$

We can represent the last integral in the form of a sum over all the balls:

$$
\mathbf{E}^{a}\left(\mathbf{r}^{\prime}, \omega\right)=\sum_{n} \iiint_{D_{(n)}} \widehat{\mathbf{G}}_{E}\left(\mathbf{r}^{\prime} \mid \mathbf{r}, \omega\right) \cdot \Delta \sigma \mathbf{E}^{b}(z, \omega) d v
$$

where $D_{(n)}$ is a volume of ball number $n$.

Assuming that the distance between the balls is also much smaller than the wavelength in the background medium, $L \ll \lambda_{1}$, we can represent the integral over one ball as an integral over one cubic cell, $V_{(n)}$, with the side equal to $L$, surrounding this ball:

$$
\begin{aligned}
& \iiint_{D_{(n)}} \widehat{\mathbf{G}}_{E}\left(\mathbf{r}^{\prime} \mid \mathbf{r}, \omega\right) \cdot \Delta \sigma \mathbf{E}^{b}(z, \omega) d v \approx \\
& \frac{D_{0}}{V_{0}} \iiint_{V_{(n)}} \widehat{\mathbf{G}}_{E}\left(\mathbf{r}^{\prime} \mid \mathbf{r}, \omega\right) \cdot \Delta \sigma \mathbf{E}^{b}(z, \omega) d v,
\end{aligned}
$$

where $D_{0}$ is the volume of a ball, and $V_{0}$ is the volume of a cube: $D_{0}=$ $(4 \pi / 3) R^{3}$, and $V_{0}=L^{3}$. Therefore, we have:

$$
\mathbf{E}^{a}\left(\mathbf{r}^{\prime}, \omega\right)=\frac{4 \pi}{3}\left(\frac{R}{L}\right)^{3} \sum_{n, m} \sum_{p} \iiint_{V_{(n, m, p)}} \widehat{\mathbf{G}}_{E}\left(\mathbf{r}^{\prime} \mid \mathbf{r}, \omega\right) \cdot \Delta \sigma \mathbf{E}^{b}(z, \omega) d v=
$$




$$
\frac{4 \pi}{3}\left(\frac{R}{L}\right)^{3} \Delta \sigma \int_{0}^{\infty}\left[\int_{-\infty}^{\infty} \int_{-\infty}^{\infty} \widehat{\mathbf{G}}_{E}\left(\mathbf{r}^{\prime} \mid \mathbf{r}, \omega\right) d x d y\right] \cdot \mathbf{E}^{b}(z, \omega) d z .
$$

Note that, in a homogeneous medium:

$$
\widehat{\mathbf{G}}_{E}\left(\mathbf{r}^{\prime} \mid \mathbf{r}, \omega\right)=i \omega \mu\left(\widehat{\mathbf{I}}+\frac{1}{k_{1}^{2}} \nabla^{\prime} \nabla^{\prime}\right) G_{0}\left(\mathbf{r}^{\prime} \mid \mathbf{r}, \omega\right),
$$

where:

$$
G_{0}=\frac{e^{i k_{1}\left|\mathbf{r}^{\prime}-\mathbf{r}\right|}}{4 \pi\left|\mathbf{r}^{\prime}-\mathbf{r}\right|}
$$

Therefore:

$$
\begin{gathered}
\mathbf{E}^{a}\left(\mathbf{r}_{j}, \omega\right)= \\
\frac{1}{3}\left(\frac{R}{L}\right)^{3} i \omega \mu \Delta \sigma\left(\widehat{\mathbf{I}}+\frac{1}{k_{1}^{2}} \nabla^{\prime} \nabla^{\prime}\right) \int_{0}^{\infty}\left[\int_{-\infty}^{\infty} \int_{-\infty}^{\infty} \frac{e^{i k_{1}\left|\mathbf{r}^{\prime}-\mathbf{r}\right|}}{\left|\mathbf{r}^{\prime}-\mathbf{r}\right|} d x d y\right] \cdot \mathbf{E}^{b}(z, \omega) d z .
\end{gathered}
$$

The integral over $X$ and $Y$ is a tabulated one:

$$
\int_{-\infty}^{\infty} \int_{-\infty}^{\infty} \frac{e^{i k_{1}\left|\mathbf{r}^{\prime}-\mathbf{r}\right|}}{\left|\mathbf{r}^{\prime}-\mathbf{r}\right|} d x d y=i 2 \pi e^{i k_{1}\left|z-z^{\prime}\right|} / k_{1}
$$

Thus, we have:

$$
\begin{gathered}
\mathbf{E}^{a}\left(\mathbf{r}^{\prime}, \omega\right)= \\
\frac{i 2 \pi}{3 k_{1}}\left(\frac{R}{L}\right)^{3} i \omega \mu \Delta \sigma\left[\left(\widehat{\mathbf{I}}+\frac{1}{k_{1}^{2}} \nabla^{\prime} \nabla^{\prime}\right) \int_{0}^{\infty} e^{i k_{1}\left|z-z^{\prime}\right|} e^{i k_{1} z} d z\right] \cdot \mathbf{b}= \\
\mathbf{b} \frac{i 2 \pi}{3 k_{1}}\left(\frac{R}{L}\right)^{3} i \omega \mu \Delta \sigma \int_{0}^{\infty} e^{i k_{1}\left|z-z^{\prime}\right|} e^{i k_{1} z} d z+\frac{i 2 \pi}{3 k_{1}^{3}}\left(\frac{R_{0}}{L}\right)^{3} i \omega \mu \Delta \sigma \boldsymbol{\nabla}^{\prime}(\mathbf{a} \cdot \mathbf{b}),
\end{gathered}
$$

where we introduced a notation:

$$
\mathbf{a}=\nabla^{\prime} \int_{0}^{\infty} e^{i k_{1}\left|z-z^{\prime}\right|} e^{i k_{1} z} d z
$$

Note that vector a contains only one nonzero component: $\mathbf{a}=\left(0,0, a_{z}\right)$, while vector $\mathbf{b}=\left(b_{x}, b_{y}, 0\right)$; therefore $(\mathbf{a} \cdot \mathbf{b})=0$, and

$$
\mathbf{E}^{a}\left(\mathbf{r}^{\prime}, \omega\right)=\mathbf{E}^{a}\left(z^{\prime}, \omega\right)=\mathbf{b} \frac{i 2 \pi}{3 k_{1}}\left(\frac{R}{L}\right)^{3} i \omega \mu \Delta \sigma \int_{0}^{\infty} e^{i k_{1}\left|z-z^{\prime}\right|} e^{i k_{1} z} d z
$$

The integral in formula (3.13) is calculated as:

$$
\int_{0}^{\infty} e^{i k_{1}\left|z-z^{\prime}\right|} e^{i k_{1} z} d z=e^{i k_{1} z^{\prime}}\left(z^{\prime}-\frac{1}{2 i k_{1}}\right)
$$

Substituting (3.14) into (3.13), we find:

$$
\mathbf{E}^{a}\left(z^{\prime}, \omega\right)=-\mathbf{E}^{b}\left(z^{\prime}, \omega\right) \frac{2 \pi}{3 k_{1}}\left(\frac{R}{L}\right)^{3} \omega \mu \Delta \sigma\left(z^{\prime}-\frac{1}{2 i k_{1}}\right) .
$$


In particular, at the depth $z^{\prime}=L$ :

$$
\mathbf{E}^{a}(L, \omega)=-\mathbf{E}^{b}(L, \omega) \frac{2 \pi}{3 k_{1}}\left(\frac{R}{L}\right)^{3} \omega \mu \Delta \sigma\left(L-\frac{1}{2 i k_{1}}\right),
$$

and at $z^{\prime}=0$ :

$$
\mathbf{E}^{a}(0, \omega)=-\mathbf{b} \frac{\pi}{3}\left(\frac{R}{L}\right)^{3} \frac{\Delta \sigma}{\sigma_{1}} .
$$

Comparing (3.15) and (3.16), we find:

$$
\mathbf{E}^{a}(L, \omega)=\mathbf{E}^{a}(0, \omega) e^{i k_{1} L}\left(1-2 i k_{1} L\right) .
$$

The total field is equal:

$$
\begin{gathered}
\mathbf{E}(L, \omega)=\mathbf{E}^{a}(L, \omega)+\mathbf{E}^{b}(L, \omega)= \\
\mathbf{b} e^{i k_{1} L}\left[1-\frac{\pi}{3}\left(\frac{R}{L}\right)^{3} \frac{\Delta \sigma}{\sigma_{1}}\left(1-2 i k_{1} L\right)\right] .
\end{gathered}
$$

On the boundary, $z=0$, the total field is found to be:

$$
\begin{gathered}
\mathbf{E}(0, \omega)=\mathbf{E}^{a}(0, \omega)+\mathbf{E}^{b}(0, \omega)= \\
\mathbf{b}\left[1-\frac{\pi}{3}\left(\frac{R}{L}\right)^{3} \frac{\Delta \sigma}{\sigma_{1}}\right] .
\end{gathered}
$$

Comparing (3.18) and (3.17), we find:

$$
\mathbf{E}(L, \omega)=\mathbf{E}(0, \omega) e^{i k_{1} L} q(\omega),
$$

where:

$$
q(\omega)=\left[1-\frac{\pi}{3}\left(\frac{R}{L}\right)^{3} \frac{\Delta \sigma}{\sigma_{1}}\left(1-2 i k_{1} L\right)\right] /\left[1-\frac{\pi}{3}\left(\frac{R}{L}\right)^{3} \frac{\Delta \sigma}{\sigma_{1}}\right] .
$$

If we substitute for the original composite model a homogeneous conductive medium with the effective conductivity $\sigma_{e f}$, then:

$$
\mathbf{E}(L, \omega)=\mathbf{E}(0, \omega) e^{i k_{e f} L},
$$

where:

$$
k_{e f}=\sqrt{i \omega \mu \sigma_{e f}}
$$

By comparing (3.21) and (3.19), we can write:

$$
e^{i k_{e f} L}=e^{i k_{1} L} q(\omega) .
$$

Taking the logarithm of both sides of the last equation, we obtain:

$$
k_{e f}=\sqrt{i \omega \mu \sigma_{e f}}=k_{1}-\frac{i}{L} \ln q(\omega) .
$$


Therefore, we obtain the following analytical expression for the effective conductivity $\sigma_{e f}$ :

$$
\sigma_{e f}=\frac{i}{\omega \mu L^{2}}\left[i k_{1} L+\ln q(\omega)\right]^{2} .
$$

Substituting (3.20) into (3.24), we have:

$$
\begin{gathered}
\sigma_{e f}(l)= \\
\sigma_{1}\left\{1-\frac{1}{2 \pi(1-i) l} \ln \left[1-\frac{\frac{\pi}{3} \nu^{3} p(4 \pi(1-i) l)}{1-\frac{\pi}{3} \nu^{3} p}\right]\right\}^{2},
\end{gathered}
$$

where:

$$
\begin{gathered}
\Delta \sigma / \sigma_{1}=\sigma_{2} / \sigma_{1}-1=p, \\
\frac{R}{L}=\nu, \text { and } \frac{L}{\lambda_{1}}=l .
\end{gathered}
$$

Note that $l$ is proportional to the square root of the frequency:

$$
l=\frac{L}{\lambda_{1}}=\frac{L}{2 \pi \sqrt{2}} \sqrt{\omega \mu_{0} \sigma_{1}}=\frac{L}{2 \pi \sqrt{2}} \sqrt{\omega \mu_{0} \sigma_{1}} .
$$

Figures (3-7) and (3-8) show the real and imaginary parts of the effective conductivity as the functions of parameter $l$ for a different conductivity contrast $p$. Taking into account formula (3.26), we can see that plots (3-7) and (3-8) represent actually the frequency dependance of the effective conductivity.

We can make several important observations by analyzing expression (3.25) for effective conductivity. First of all, it has a complex value and it depends on frequency, while the original conductivities, $\sigma_{1}$ and $\sigma_{2}$, were real and frequencyindependent constants. This fact means that one would observe the IP effect by conducting the electrical measurement over a composite medium formed by the materials with the real conductivities.

Second, the parameters $p, \nu$, and $l$ are determined by the intrinsic physical and geometrical characteristics of the composite medium: the conductivity contrast between the different phases of the medium, $\sigma_{2} / \sigma_{1}$, the radius $R$ of spherical inclusions, and the closest distance, $L$, between their centers. Therefore, in principle, the effective conductivity formula (3.25) may serve as a basis

for determining the intrinsic characteristics of the polarizable rock formation from the observed electrical data. Future research in the framework of this project will be focused on developing the methods of solving this problem.

\subsubsection{Asymptotics of the effective conductivity}

Consider the case when $l \ll 1$. Taking into account that:

$$
\ln (1+x) \approx x,
$$




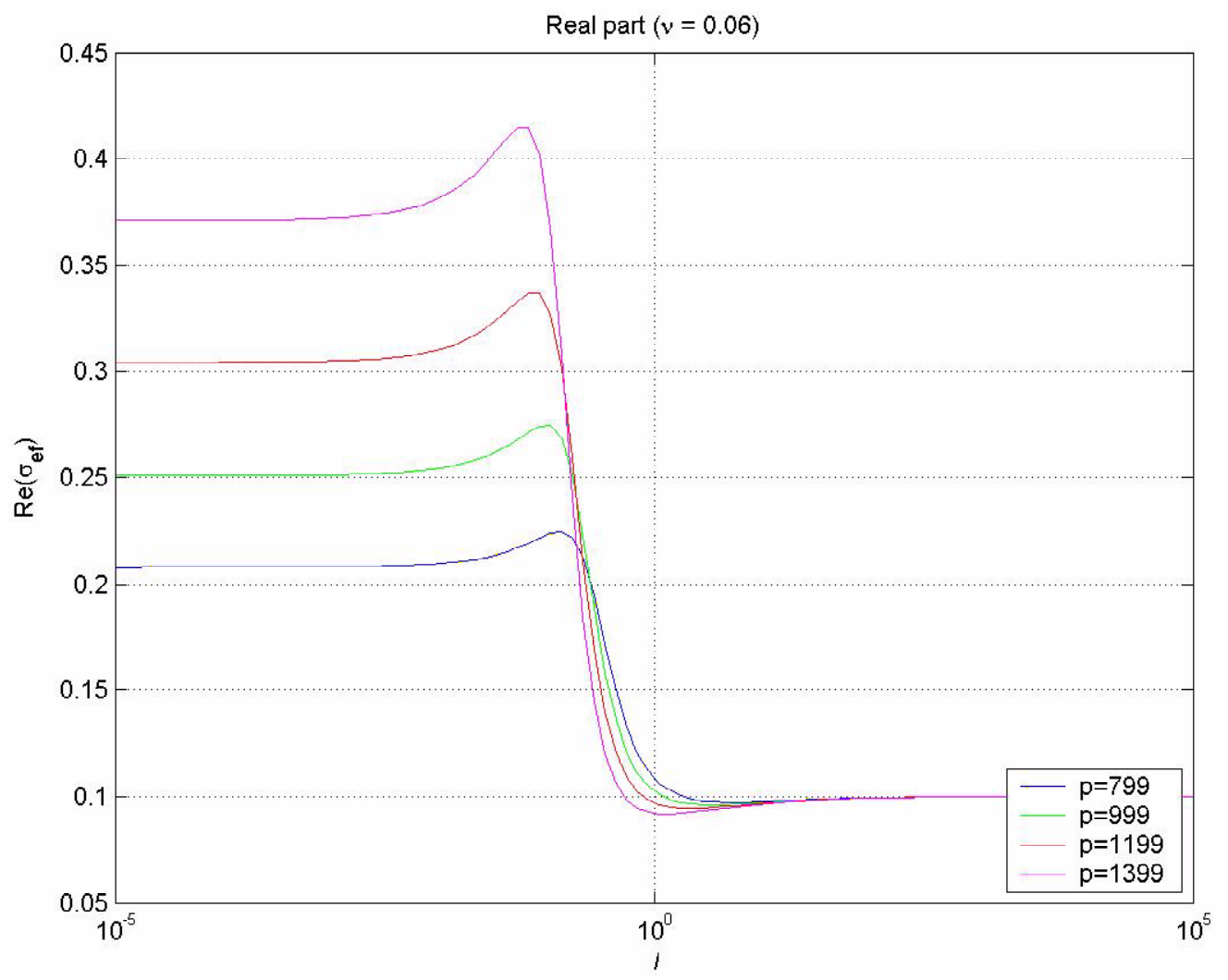

Figure 3-7 The real part of the effective conductivity. The conductivity contrast, $p$, is the code of the curves. 


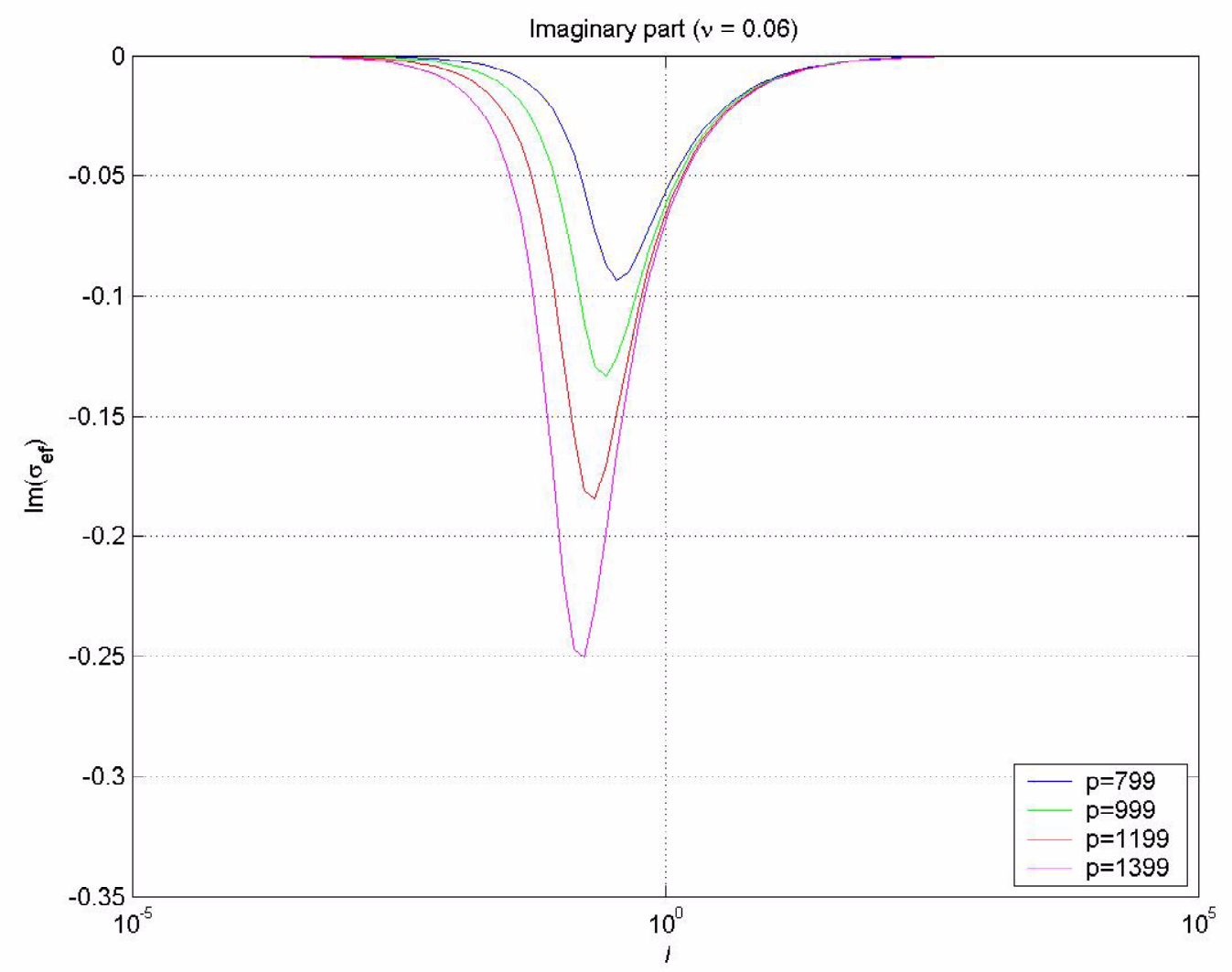

Figure 3-8 The imaginary part of the effective conductivity. The conductivity contrast, $p$, is the code of the curves. 
we write:

$$
\sigma_{e f}(l) \approx \sigma_{1}\left\{1+\frac{1}{2 \pi(1-i) l} \cdot \frac{\frac{\pi}{3} \nu^{3} p 4 \pi(1-i) l}{\left(1-\frac{\pi}{3} \nu^{3} p\right)}\right\}^{2}=\sigma_{1}\left\{\frac{1+\frac{\pi}{3} \nu^{3} p}{1-\frac{\pi}{3} \nu^{3} p}\right\}^{2} .
$$

From the last formula, for small inclusions and a relatively small conductivity contrast, $\frac{\pi}{3} \nu^{3} p \ll 1$, we have:

$$
\sigma_{e f}(l) \approx \sigma_{1}, \text { if } l \rightarrow 0
$$

For large $l \gg 1$, taking into account that

$$
\frac{\ln (1+x)}{x} \rightarrow 0, \text { if } x \rightarrow \infty
$$

we find again

$$
\sigma_{e f}(l) \approx \sigma_{1}, \text { if } l \rightarrow \infty .
$$

We should note in the conclusion of this section that the developed method of constructing a mathematical model of the IP effect based on the effectivemedium theory can be extended to the multi-phases heterogeneous conductive medium, which will consitute a subject of future research. 


\section{EXPERIMENTAL}

\subsection{Development of the 3-D EM-IP forward mod- eling system}

For modeling an IP response in the frequency domain, i.e., spectral IP modeling, we need to solve the Maxwell's equations for the complex conductivity model. Using this modeling method, we can investigate the effect of different complex conductivity relaxation models, including the Cole-Cole model and a composite conductivity model, and we can obtain the EM responses for the various types of transmitting sources including the current bipoles used in conventional IP surveys.

During the first year of the project we have developed a prototype of the 3-D IP modeling algorithm using the integral equation (IE) method. Our IE forward modeling code INTEM3DIP is based on the contraction IE method, which improves the convergence rate of the iterative solvers (Hursán and Zhdanov, 2002). This code can handle various types of sources and receivers to compute the effect of the complex resistivity model. In addition, the preprocessing and postprocessing routines are developed to obtain conventional IP responses as well. We have tested the working version of the INTEM3DIP code for computer simulation of the IP data for several models including the southwest US porphyry model and the Kambalda-style nickel sulfide deposit in Australia. The results of this modeling study are presented below.

\subsection{Application of INTEM3DIP to the southwest U.S. porphyry model}

Figure 4-1 shows a resistivity/IP model of a "typical" porphyry copper system in the southwestern U. S. This model is characterized by potentially strong EM coupling as well as IP effects. We performed various modeling experiments to get an insight into the IP responses for this model. At first, we ran this model as a layered case, and then moved to 3-D models, which were simplified versions of the conceptual porphyry deposit model presented in Figure 4-1. We have used the electrical bipole sources in modeling to simulate the conventional IP responses, but, in principle, the INTEM3DIP software makes it possible to 


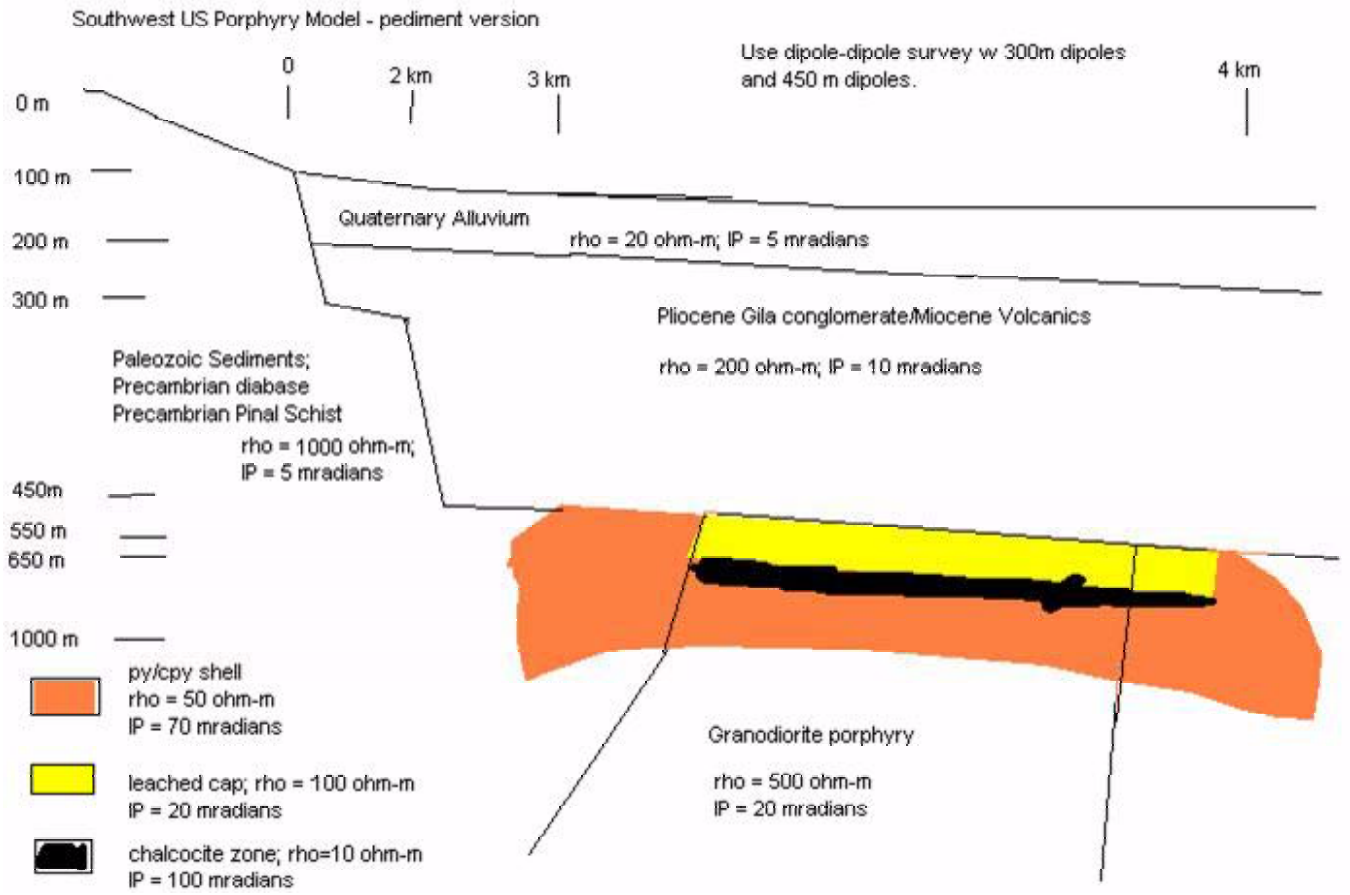

Figure 4-1 A resistivity/ip model of a "typical" porphyry copper system in the southwestern U.S.

investigate the effects of complex resistivity using various types of sources.

\subsubsection{1-D Model}

We start with the simplified 1-D model shown in Figure 4-2. This model is obtained by simply extracting the conductivity values at the middle of the profile in the original model of a porphyry copper system in the southwestern U. S.

It is represented by a 6-layered earth model. The first layer corresponds to Quaternary alluvium in the original model, the second layer is Pilocene Gila conglomerate/Miocene volcanics, the third layer corresponds to the leached cap, the fourth layer is a chalcocite zone, the fifth layer describes the py/cpy shell, and the sixth layer is Granodiorite porphyry. The Paleozoic sediment in the original model, however, is omitted in this 1-D model. It will be included, however, in 3-D models 4 and 5 . Note that the sequence from the third to the fifth layers corresponds to the anomalous zone appearing in the original model. The thicknesses of layers of the 1-D model are 50, 50, 150, 150, and $100 \mathrm{~m}$, respectively. The conductivities of the layers are calculated according to this equation: 


\section{D Model}

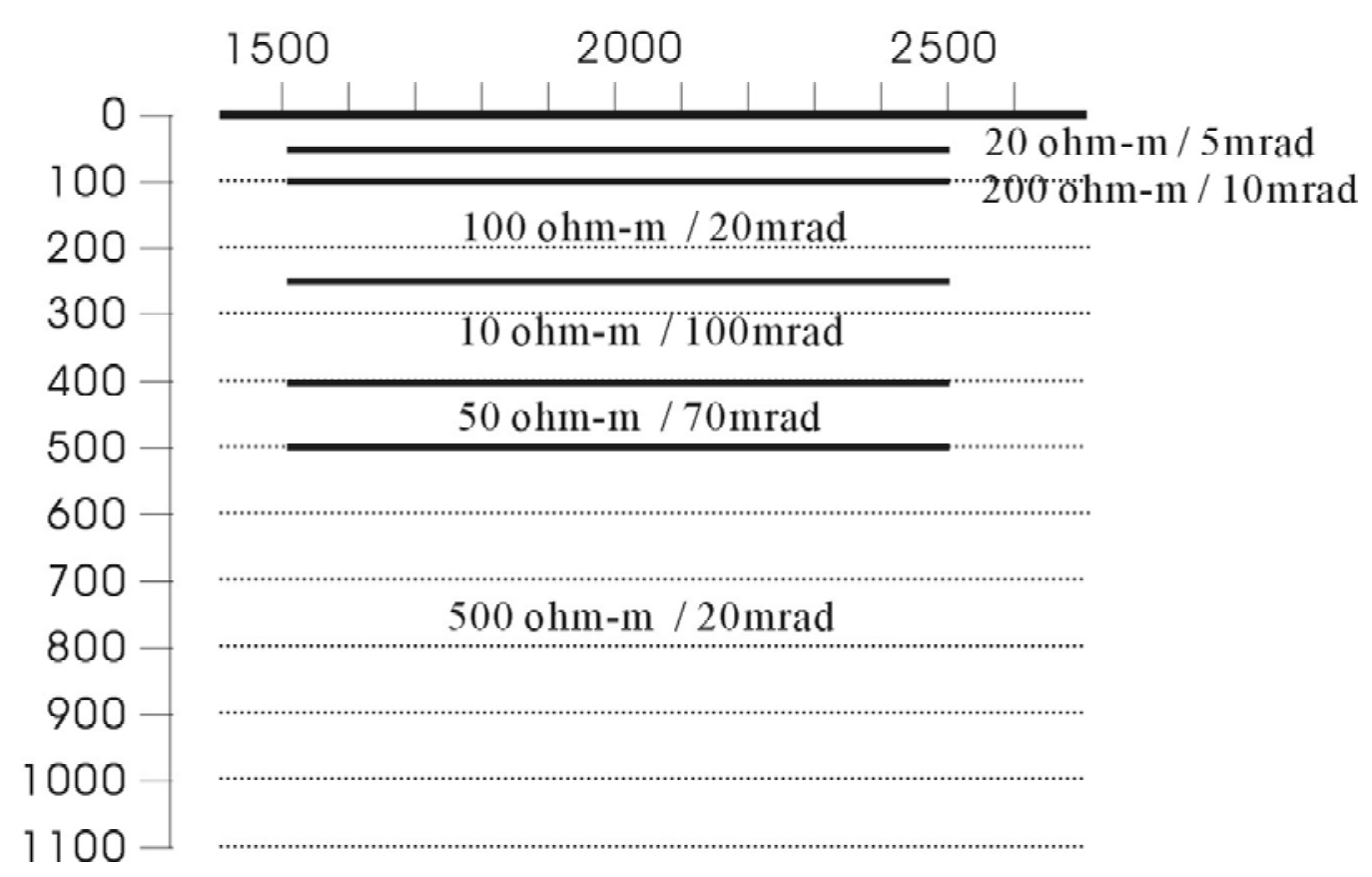

Figure 4-2 1-D model obtained from the conceptual model of a "typical" southwest U.S. porphyry deposit. 


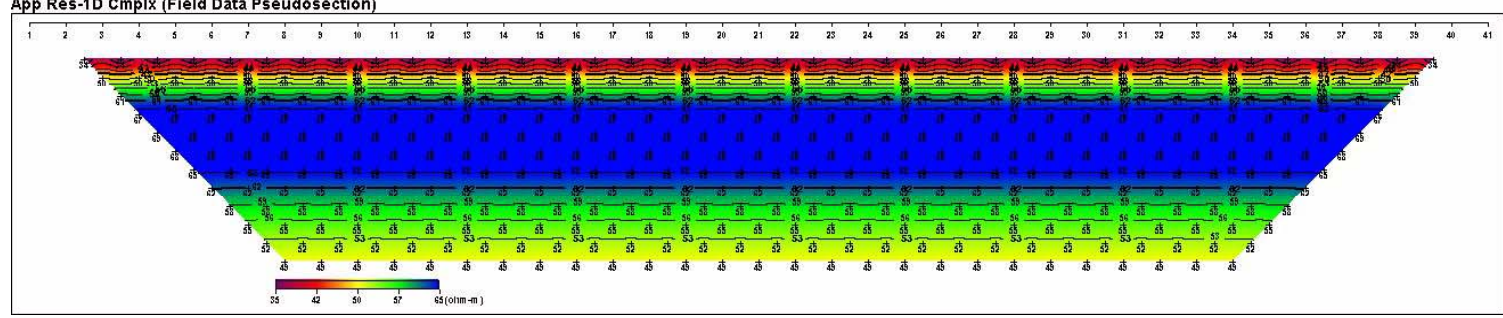

Figure 4-3 Apparent resistivity pseudosection obtained by the INTEM3DIP code for a 1-D IP model.

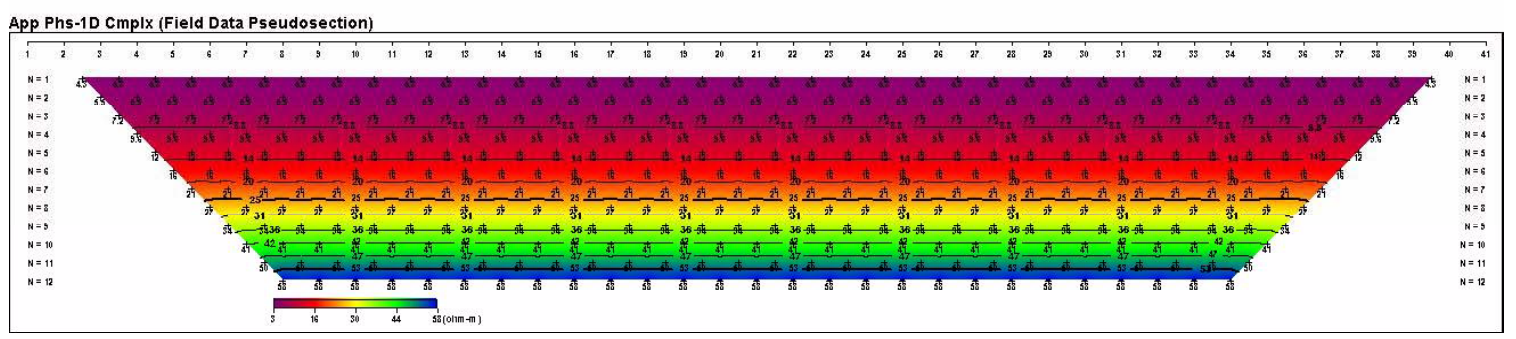

Figure 4-4 Pseudosection of the phase of apparent resistivity obtained by the INTEM3DIP code for a 1-D IP model.

$$
\sigma_{j}=\left(\frac{1}{\rho_{j}}\right) e^{i \phi_{j}}, \quad j=1, \ldots, 6,
$$

where $\rho_{j}$ are 20, 200,100,10, 50 and 500 Ohm-m and $\phi_{j}$ are 5, 10, 20, 100, 70 , and 20 mili-radians of each layer, respectively. The corresponding real and imaginary parts of the complex conductivity of each layer are $[0.05,0.005$, 0.01, 0.0995, 0.02, 0.002] Ohm-m and [0.0002, 5e-005, 0.0002, 0.01, 0.0014, and 4e-005] Ohm-m, respectively.

Modeling is done using the draft version of the INTEM3DIP code, which can handle complex conductivity. The arrays of transmitters and receivers are dipole-dipole type arrays. To simulate the IP response as a potential difference measured by a dipole receiver, we compute the electric field along the receiver dipole location and then integrate it numerically to obtain the potential difference between two ends of the receiver bipole. The frequency of source is $0.25 \mathrm{~Hz}$, which is a mid frequency among many frequencies generally available in modern IP instruments. Apparent resistivity and phase are calculated using equations (3.6)-(3.7).

Figures 4-3 and 4-4 show the apparent resistivity and phase pseudosection of the IP responses using dipole-dipole array. Figure 4-3 represents a pseudosection of the amplitude of apparent resistivity, and Figure 4-4 is a phase pseudosection. As we expect for a 1-D model, we observe 1-D layered response in the pseudosections of the apparent resistivity and phase. 


\section{D Model}

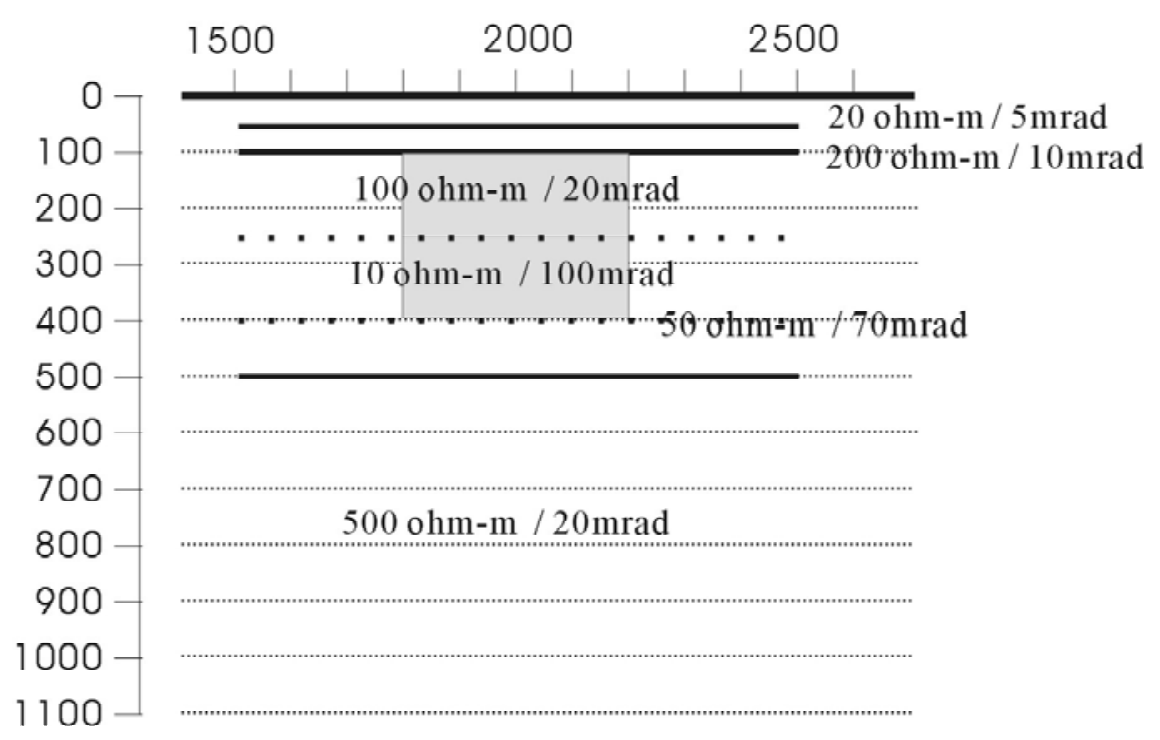

Figure 4-5 One of the 3-D models established from the conceptual model for a southwest U.S. porphyry deposit.

\subsubsection{3-D models}

One can build several different 3-D models corresponding to the conceptual resistivity/IP[ model of a "typical" porphyry copper system in the southwestern U. S., presented in Figure 4-1. One of these simplified models is shown in Figure 4-5. This 3-D Model 1 is set up by shrinking the third and the fourth layer and the fifth layer surrounding these 3-D bodies. The horizontal dimensions of two 3 -D bodies are $400 \times 400 \mathrm{~m}$ and their thicknesses are $150 \mathrm{~m}$. Then the layer thicknesses are $[50,50,400] \mathrm{m}$, and the real and imaginary parts of the conductivity are $[0.05,0.005,0.02,0.002]$ Ohm-m and [0.0002, 5e-005, 0.0014, 4e-005] Ohm-m, respevtively. The dipole-dipole survey was simulated using INTEM3DIP. The dipole length is $100 \mathrm{~m}$, and the maximum separation index $n$ is 12 .

We can identify the effect of a 3-D body in the apparent resistivity and phase pseudosection from data $n=3-5$ in Figures 4-6 and 4-7. To identify IP responses, another modeling experiment was done for the same model with the same parameters except that all conductivities of layers and the 3-D bodies are real by setting the imaginary parts at zero. The results are shown in Figures $4-8$ and $4-9$.

Figures 4-6, 4-7, 4-8 and 4-9 clearly show that apparent resistivities in both cases are the same, but a phase pseudosection shows the differences between real and complex conductivity cases. 


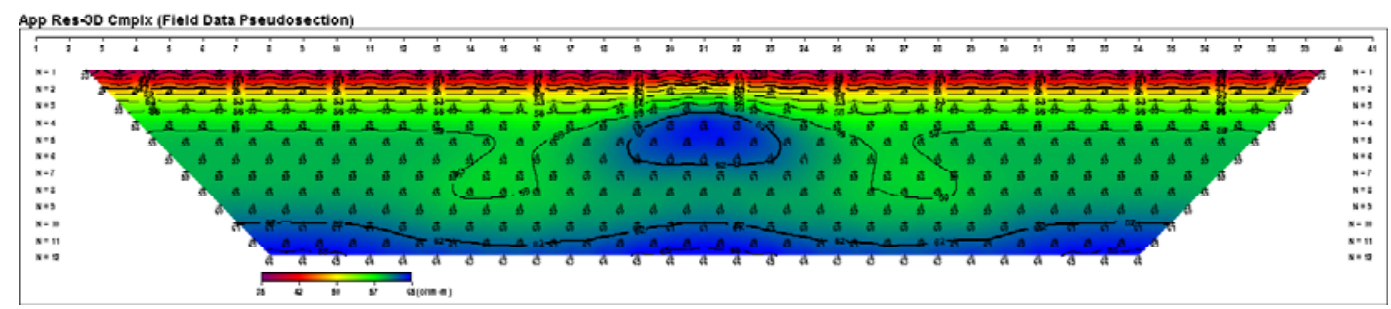

Figure 4-6 Apparent resistivity obtained by the INTEM3DIP code for 3-D Model 1.

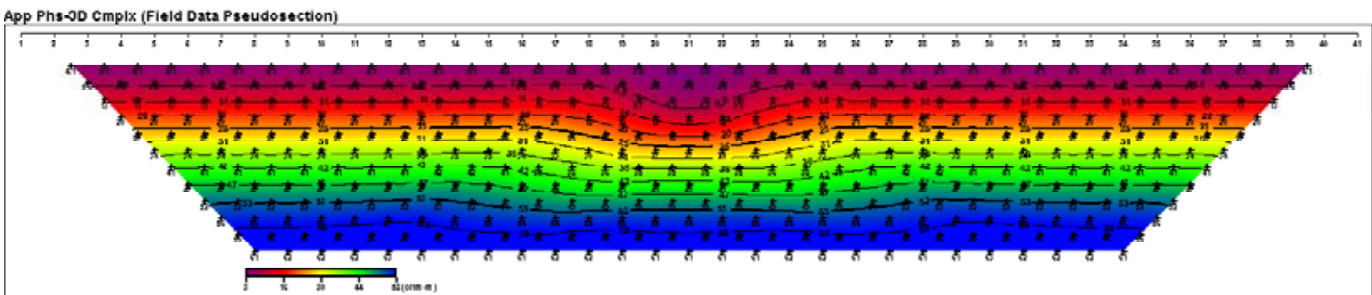

Figure 4-7 The phase of apparent resistivity obtained by the INTEM3DIP code for 3-D Model 1.

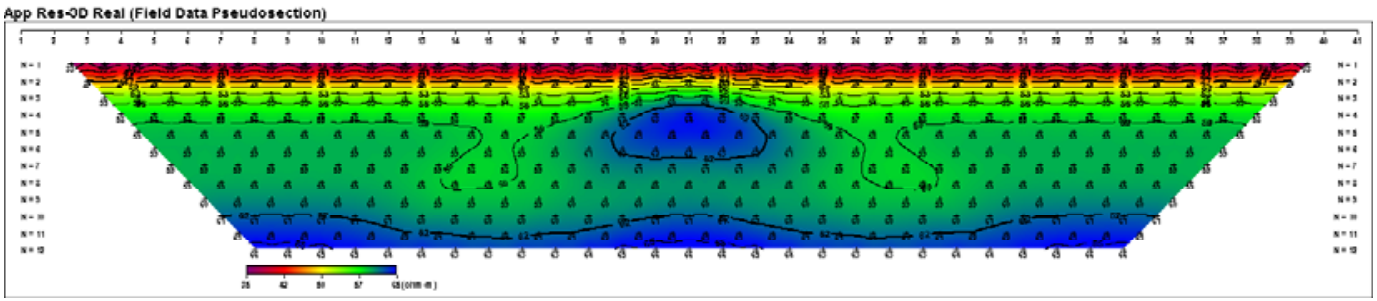

Figure 4-8 Apparent resistivity obtained by the INTEM3DIP code for 3-D Model 1. In this modeling, the imaginary part of the conductivities is set to zero to eliminate the IP effects.

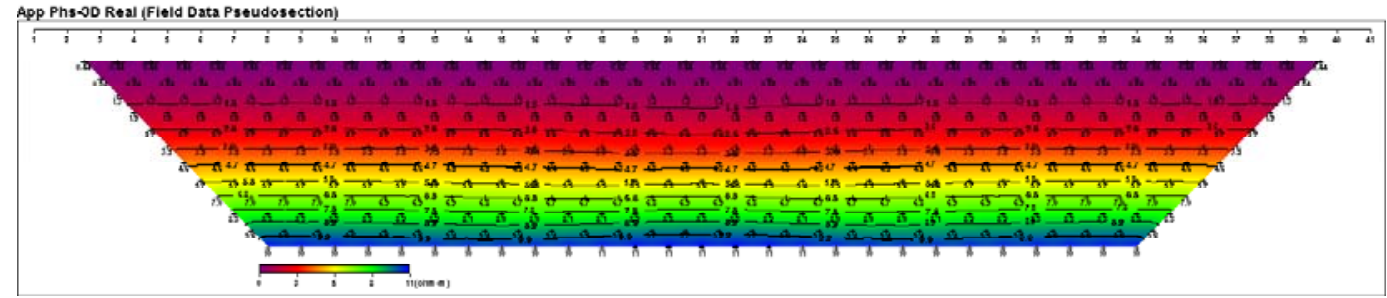

Figure 4-9 The phase of apparent resistivity obtained by the INTEM3DIP code for 3-D Model 1. In this modeling, the imaginary part of the conductivities is set to zero to eliminate the IP effects. 


\section{D Model-2}

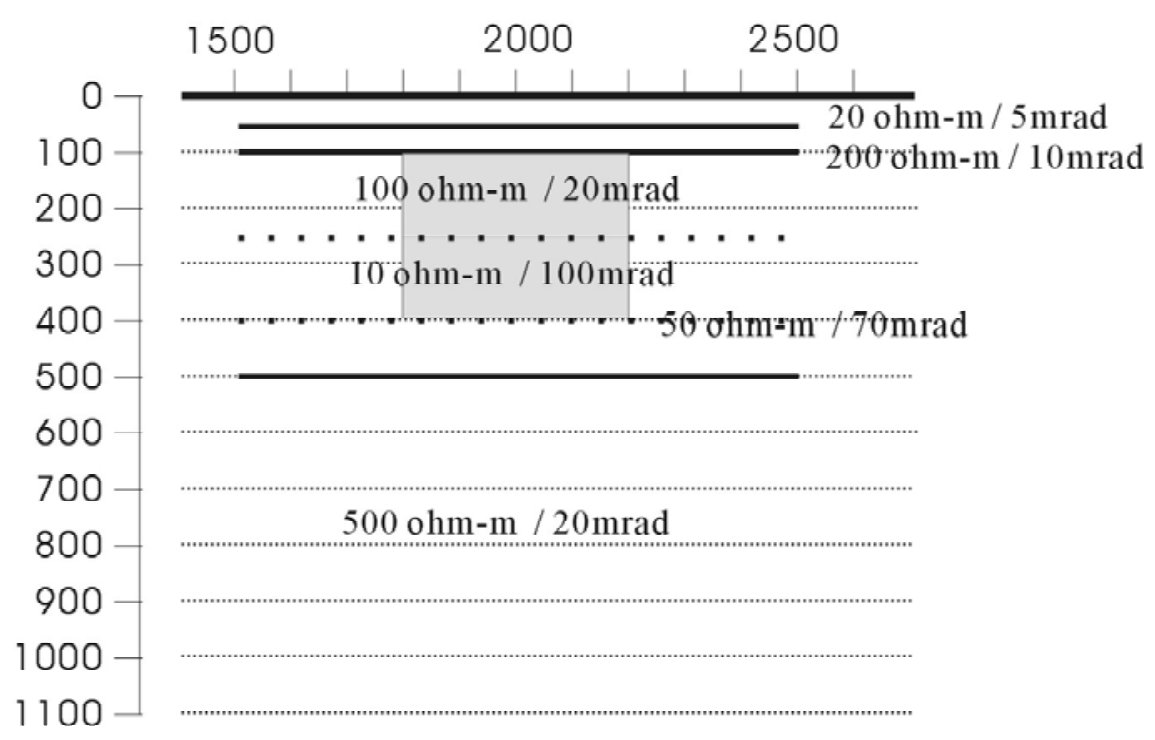

Figure 4-10 Vertical section of 3-D Model 2 of southwest U.S. porphyry deposit.

In the previous experiments, it was hard to detect the responses from deeper sections of the model. To see these effects, other experiments were performed with a longer dipole length and a larger separation index. We consider three variations of 3-D Model 1.The geoelectrical parameters of Model 2 are exactly the same as for Model 1; however, the survey parameters are different: the dipole length is of $200 \mathrm{~m}$ and the maximum separation index is equal to $n=15$. 3 -D models 3 and 4 are constructed by horizontally shrinking the fourth and fifth layers of the 1-D model, as shown in Figures 4-14 and 4-18. In this case, all modeling parameters are the same as for Model 2. The difference between Model 3 and Model 4 is that the third layer, the leached cap, is the third layer which surrounds the 3-D bodies in Model 3, but in Model 4 a 3-D body is included in the Paleozoic sediments.

The final 3-D Model 5 (Figure 4-22) is the closest to the original conceptional model of a "typical" porphyry copper system in the southwestern U. S. The leached cap, chalcocite zone, and py/cpy shell are represented in Model 5 by 3 -D bodies included in wider Paleozoic sediments.

The following figures show the pseudosections of apparent resistivity and phase computed for a dipole length of $200 \mathrm{~m}$ and the maximum separation index equal to $n=15$ for Model 2 through Model 5. We also plot the Ex component of the observed electric field normalized by the background electric field Ex for all these models. 


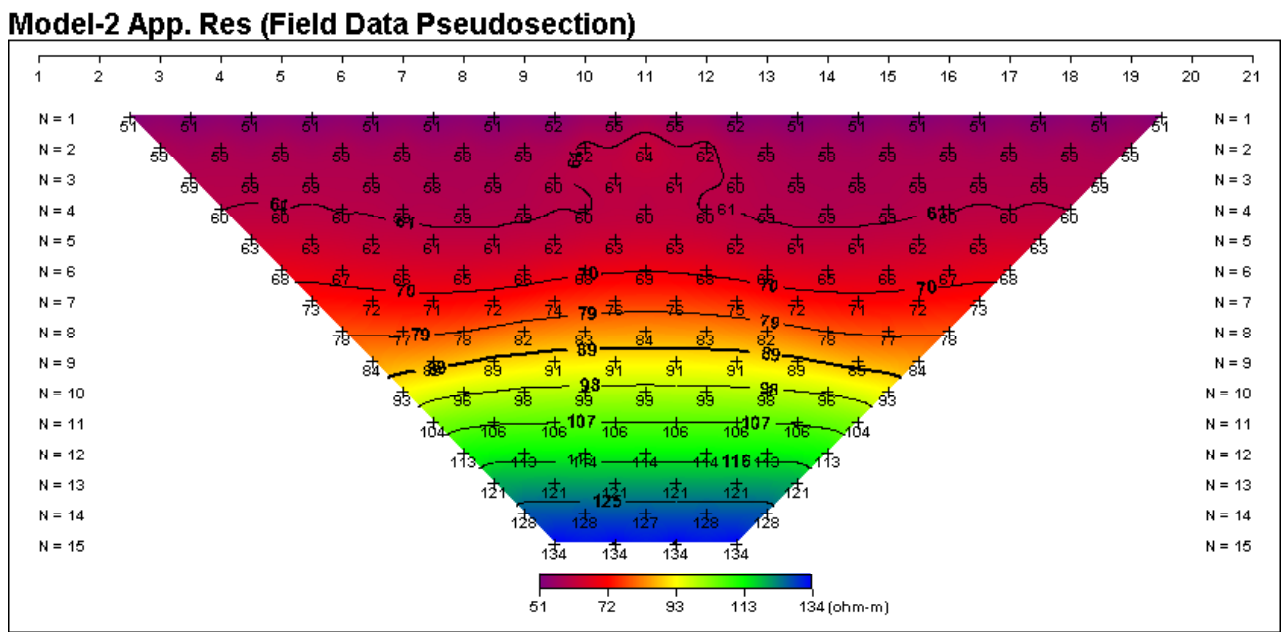

Figure 4-11 Apparent resistivity pseudosection obtained by INTEM3DIP for 3-D Model 2.

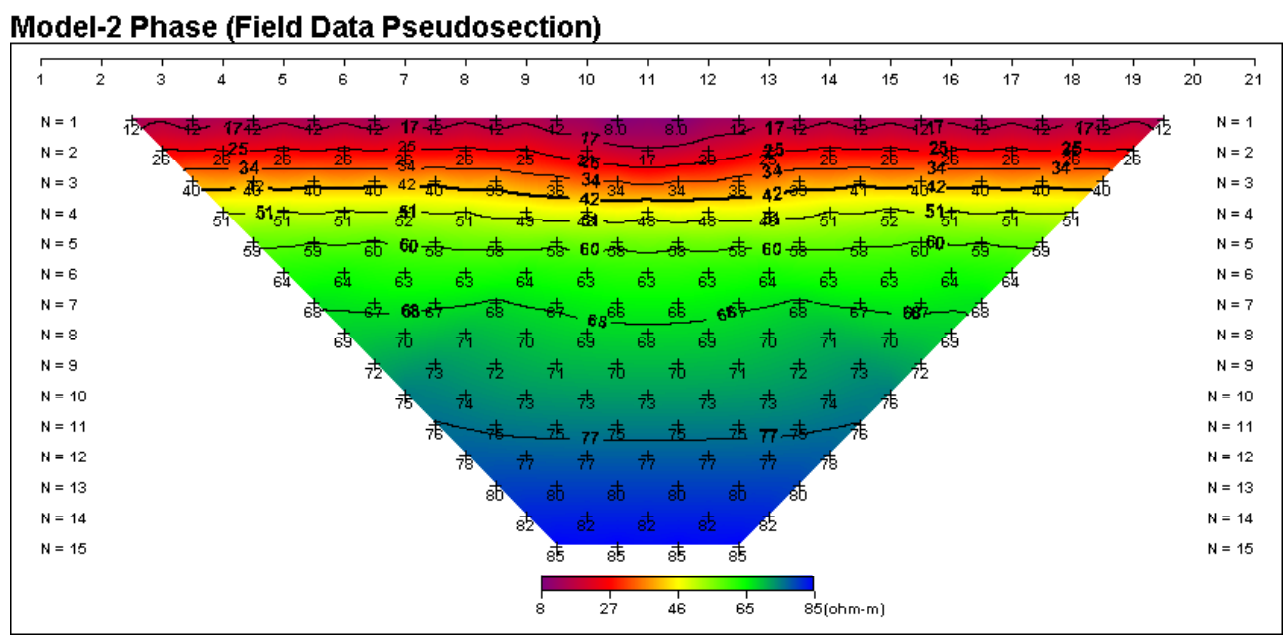

Figure 4-12 The phase pseudosection of apparent resistivity obtained by INTEM3DIP for 3-D Model 2. 

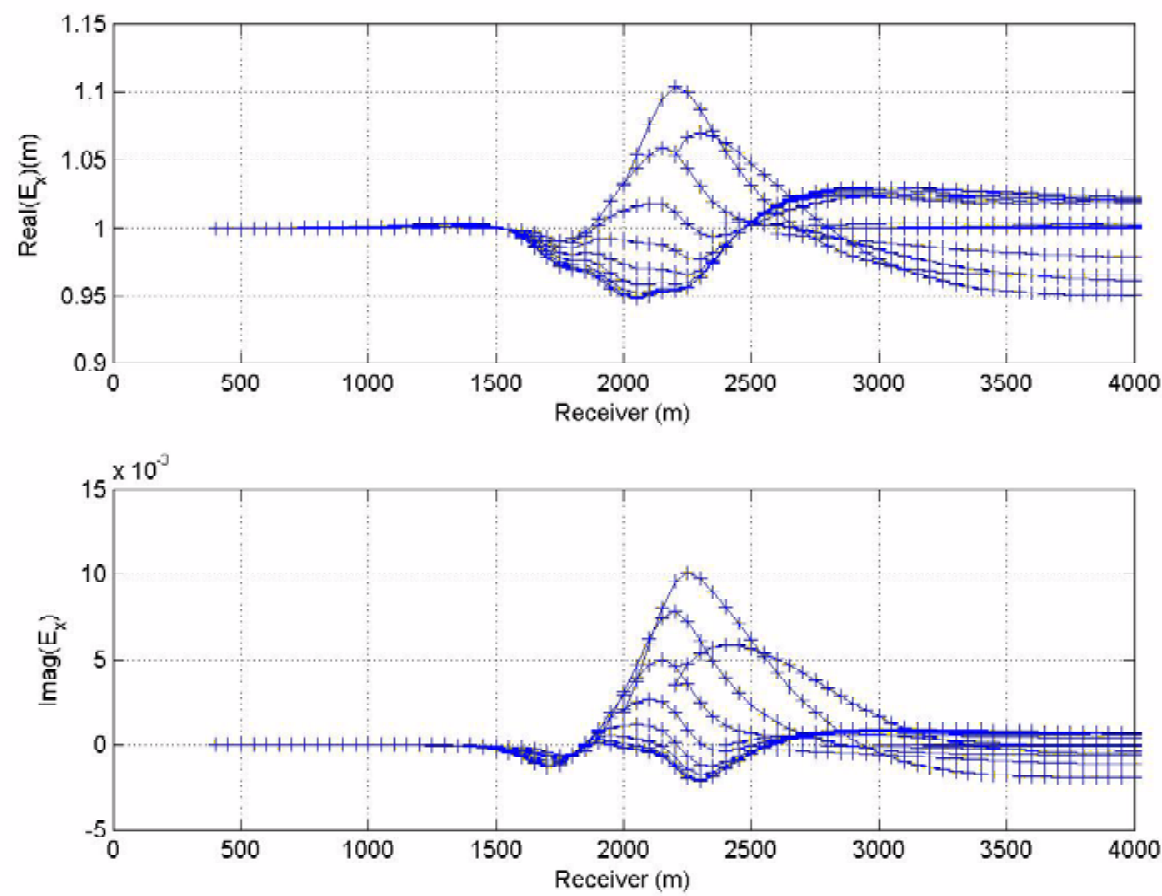

Figure 4-13 Plot of the Ex component of the observed electric field normalized by the background electric field Ex for 3-D Model 2. Each curve corresponds to one source location.

\section{D Model-3}

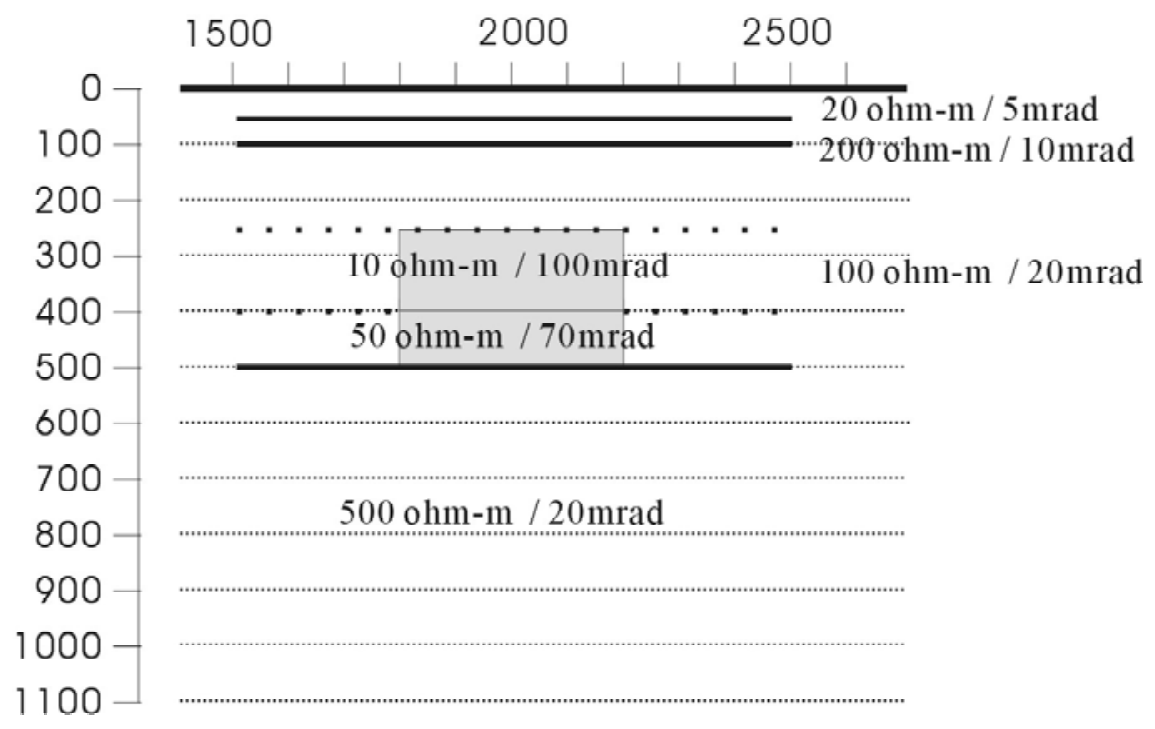

Figure 4-14 Vertical section of 3-D Model 3 of southwest U.S. porphyry deposit. 


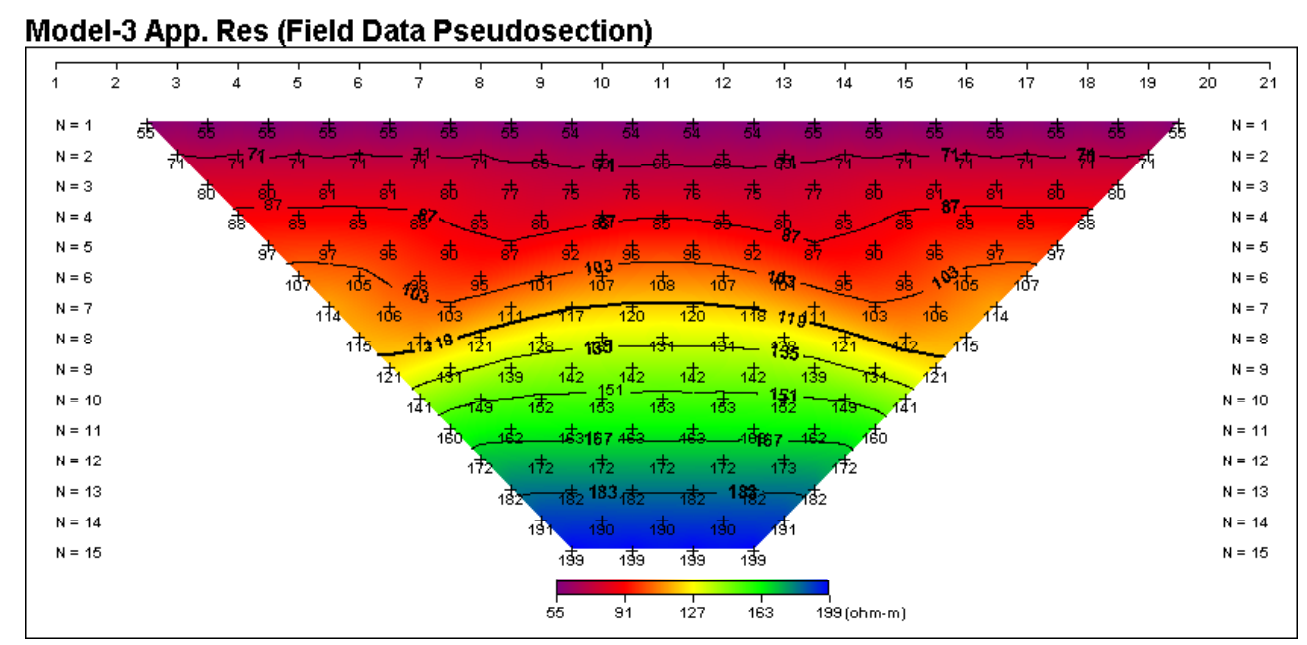

Figure 4-15 Apparent resistivity pseudosection obtained by INTEM3DIP for 3-D Model 3.

Model-3 Phase (Field Data Pseudosection)

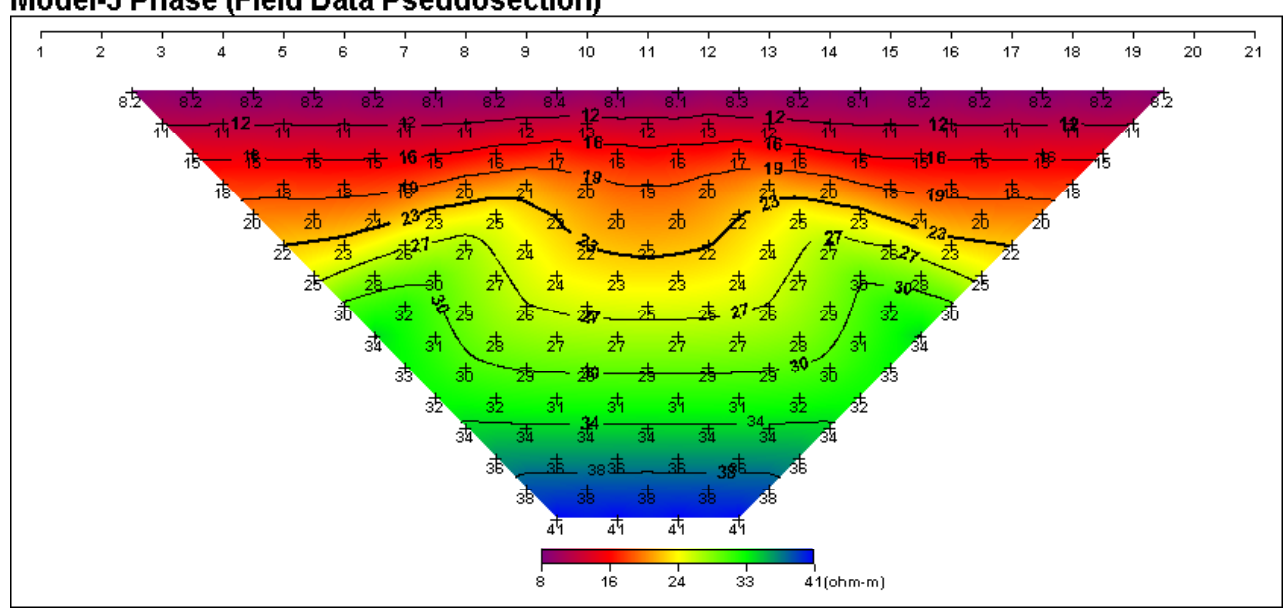

Figure 4-16 The phase pseudosection of apparent resistivity obtained by INTEM3DIP for 3-D Model 3. 

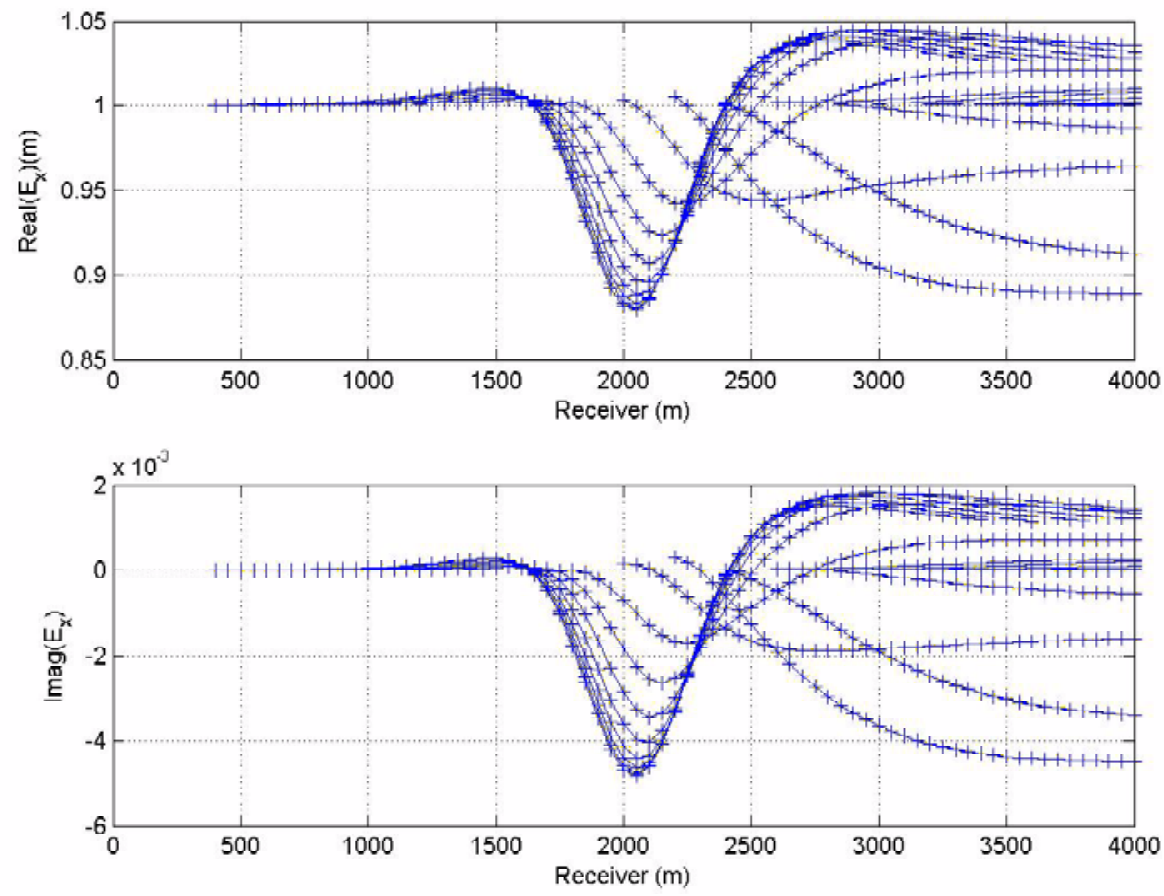

Figure 4-17 Plot of the Ex component of the observed electric field normalized by the background electric field Ex for 3D Model 3. Each curve corresponds to one source location.

\section{D Model-4}

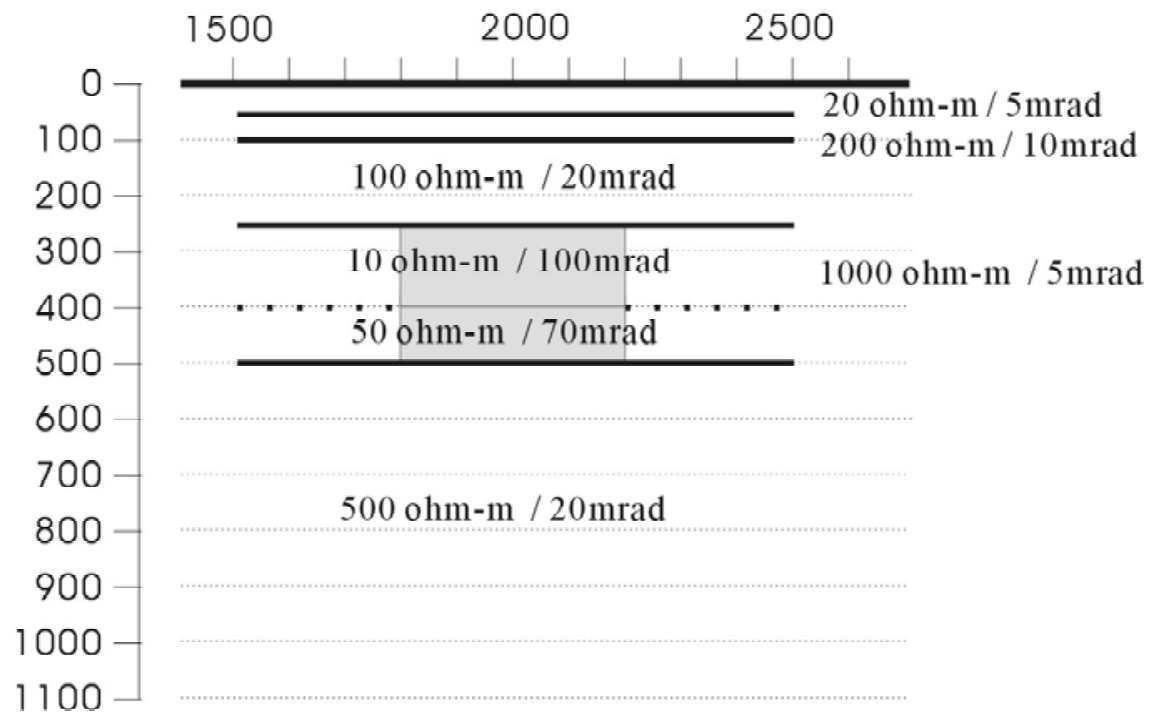

Figure 4-18 Vertical section of 3-D Model 4 of southwest U.S. porphyry deposit. 


\section{Model-4 App. Res (Field Data Pseudosection)}

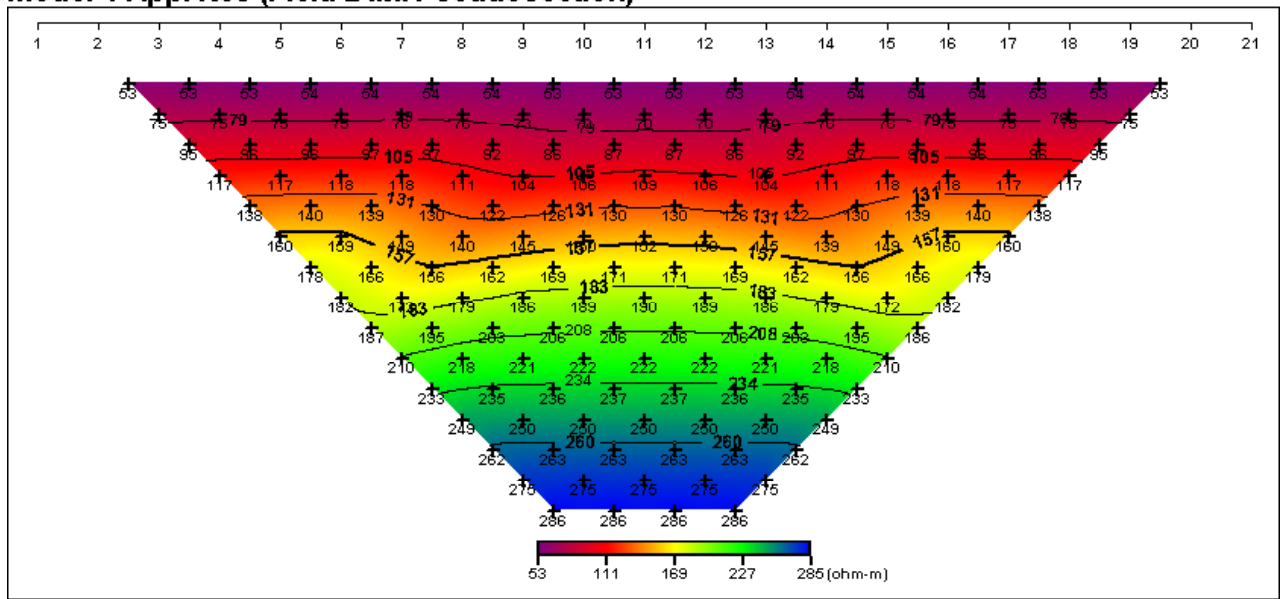

Figure 4-19 Apparent resistivity pseudosection obtained by INTEM3DIP for 3-D Model 4

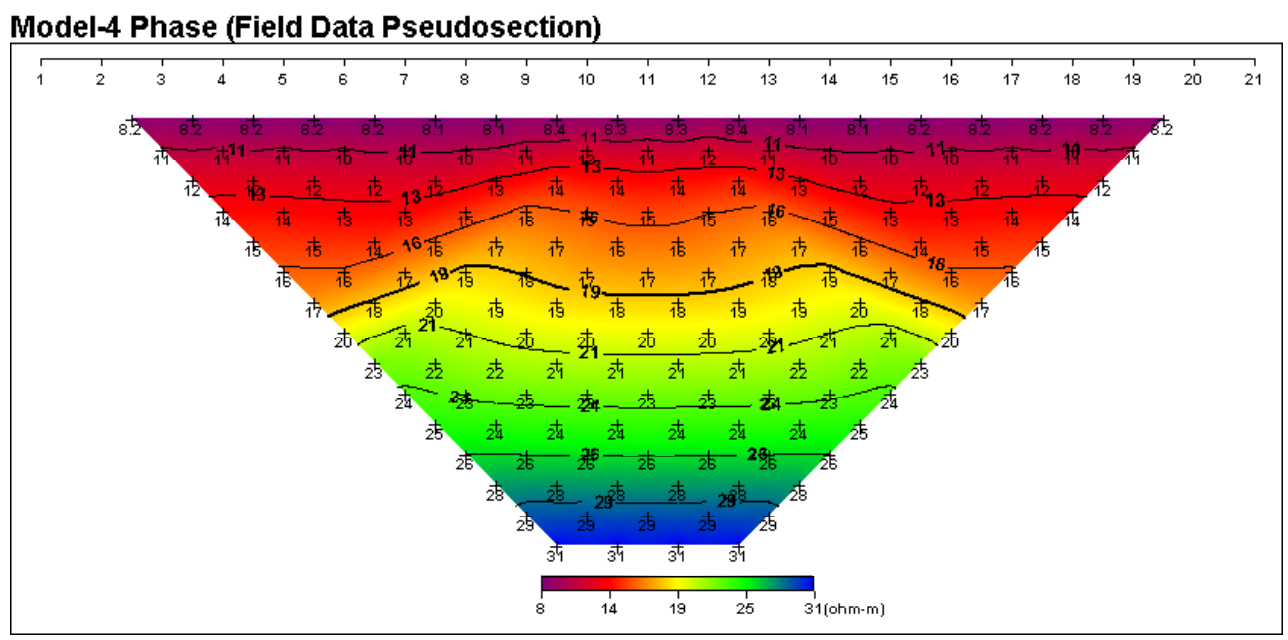

Figure 4-20 The phase pseudosection of apparent resistivity obtained by INTEM3DIP for 3-D Model 4. 

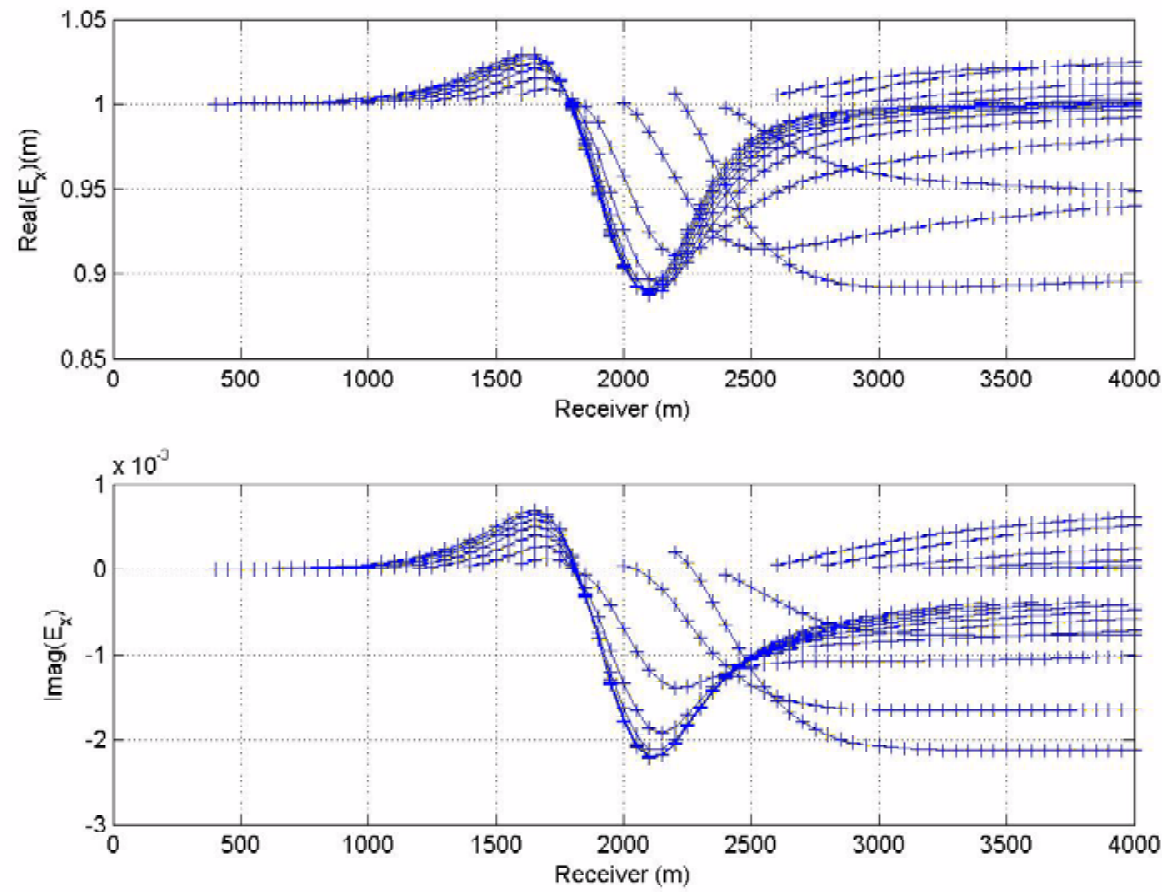

Figure 4-21 Plot of the Ex component of the observed electric field normalized by the background electric field Ex for 3-D Model 4. Each curve corresponds to one source location.

\section{D Model-5}

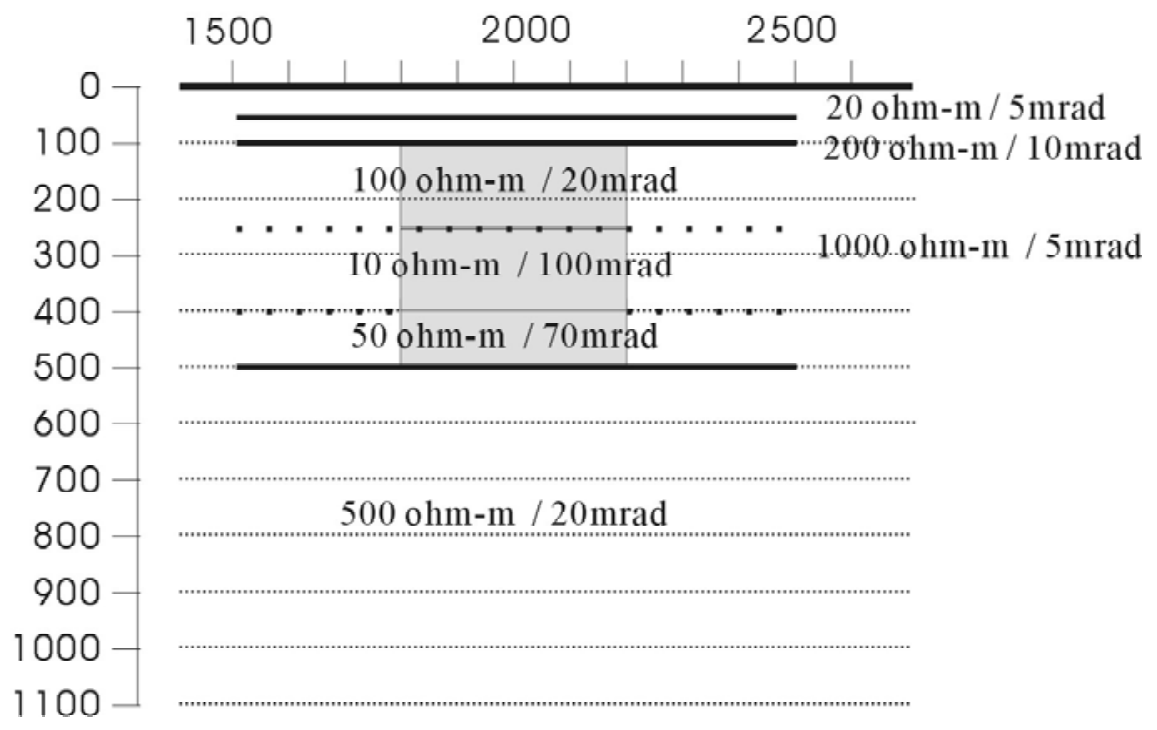

Figure 4-22 Vertical section of 3-D Model 5 of southwest U.S. porphyry deposit. 


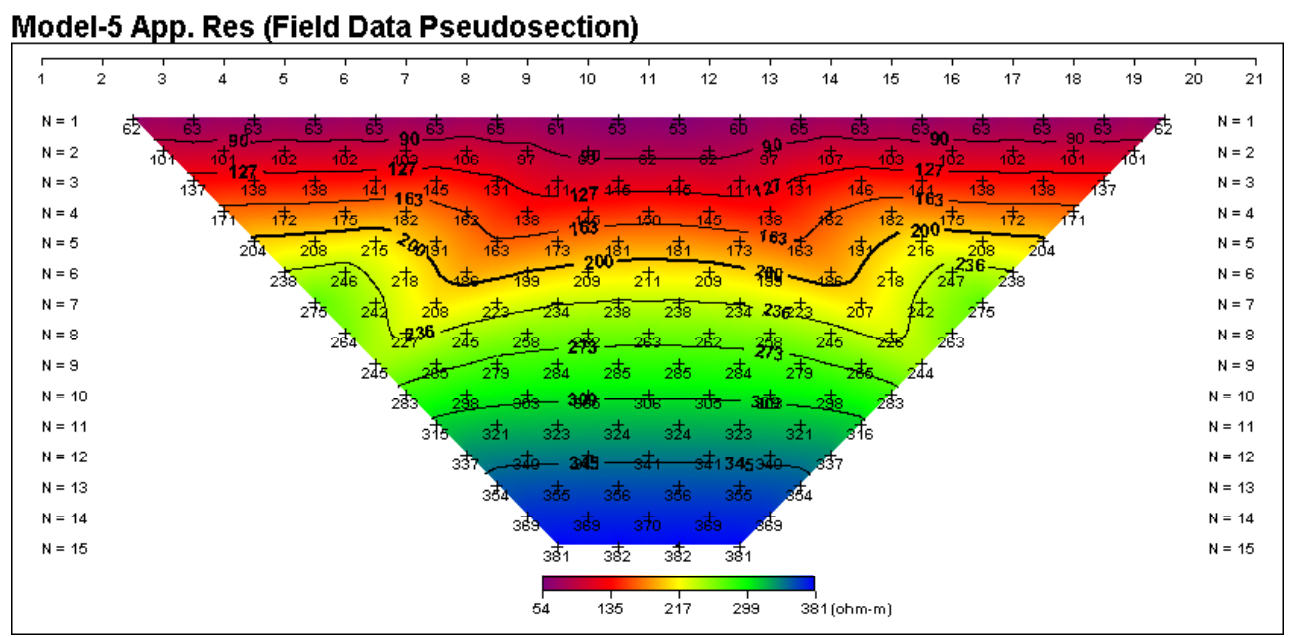

Figure 4-23 Apparent resistivity pseudosection obtained by INTEM3DIP for 3-D Model 5.

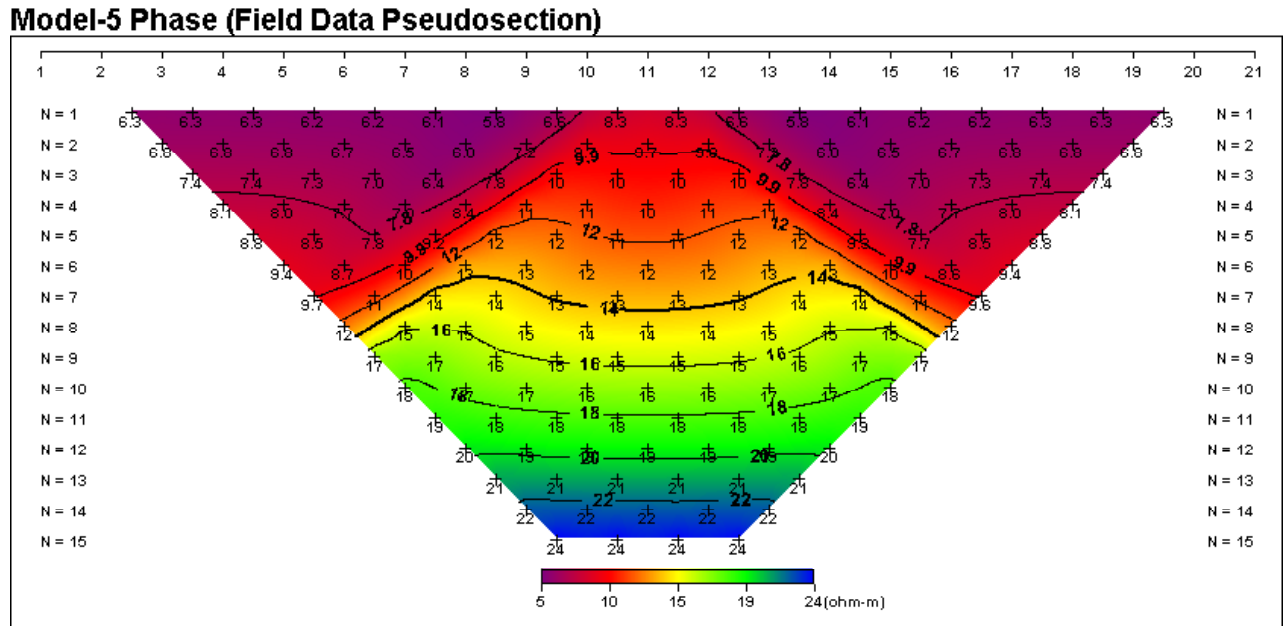

Figure 4-24 The phase pseudosection of apparent resistivity obtained by INTEM3DIP for 3-D Model 5. 

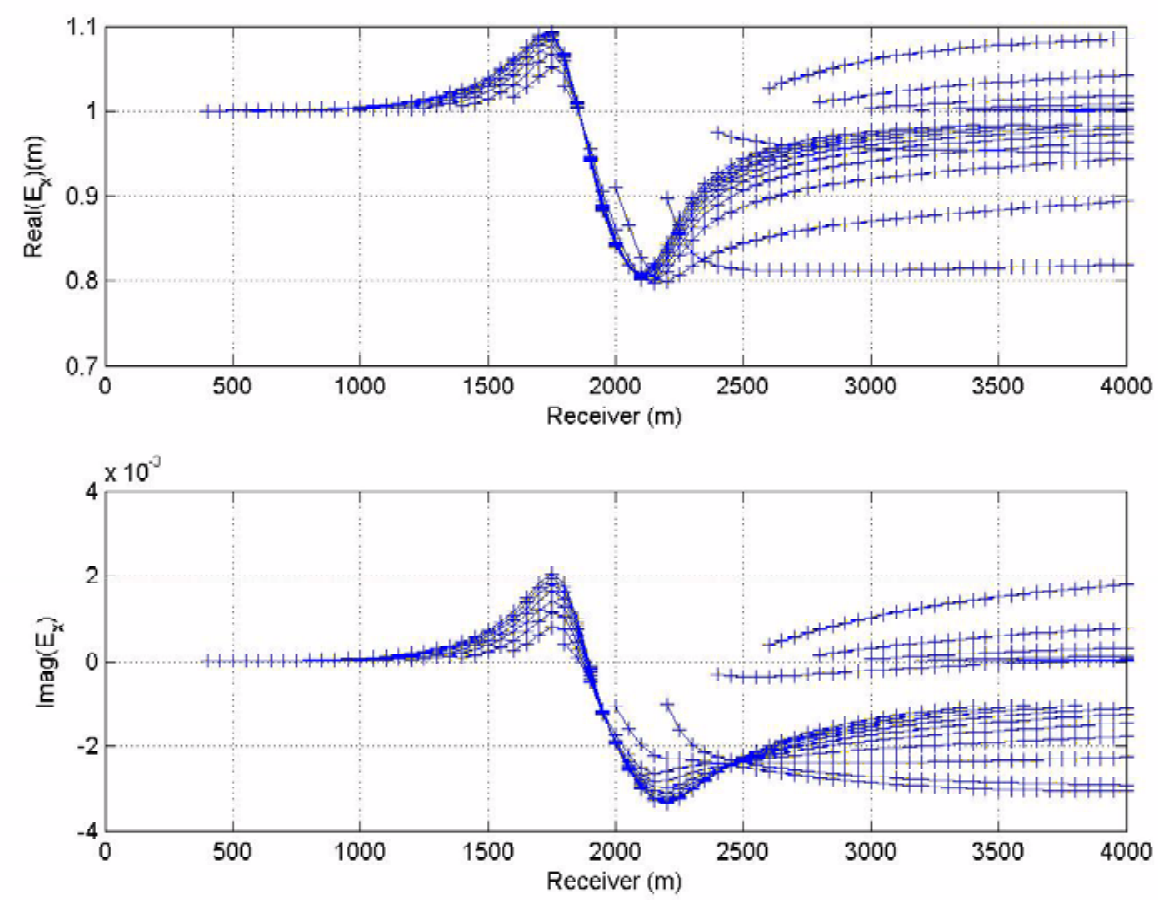

Figure 4-25 Plot of the Ex component of the observed electric field normalized by the background electric field Ex for 3-D Model 5. Each curve corresponds to one source location. 


\subsection{Application of INTEM3DIP to the model of a Kambalda-style nickel sulfide deposit}

We have computer simulated the EM-IP response for a model of the Kambaldastyle nickel sulfide deposit in Australia. In tropical and arid terrains such as Australia and southern Africa, the differential weathering characteristics of the near-surface rock units comprising the regolith often result in large lateral changes in the overburden conductivity. In the general exploration model, the regolith is highly conductive and overlays the resistive host rock and conductive target. To the explorer trying to use EM methods, this inhomogeneous regolith creates "false" anomalies due to conductive sedimentary and weathered units, and the extensive blanket of salt lake sediments in certain areas effectively masks the EM response from the target (e.g., McCracken et al., 1986). All these factors point towards the associated analysis of EM-IP responses being an extremely challenging problem.

To demonstrate the effectiveness of our modeling software, we simulated an IP survey above a stratiform komatiitic peridiotite-hosted (Type 1A), or Kambalda-style, nickel sulfide deposit located beneath a complicated regolith horizon. As described by Stone and Masterman (1998), Kambalda-style orebodies occur at, or close to, the basal contact between the lowermost komatiite flow (ultramafic) and footwall metabasalts (mafic). The NiS ore is comprised of basal massive, matrix and disseminated sulfide ores within which the nickel grade decreases upward. Strike lengths of the orebodies vary between $50 \mathrm{~m}$ and $350 \mathrm{~m}$ for mineralized widths of $5 \mathrm{~m}$ to $20 \mathrm{~m}$.

As discussed in detail by Trench and Williams (1994), potentially all geophysical techniques have application for the direct exploration of nickel sulfide (NiS) deposits. For EM methods, Kambalda-style models have been the subject of previous 3-D EM forward modeling studies such as Stolz et al. (1995) and Zhdanov et al. (2000). However, there are specific limitations on EM methods for the practical exploration of Kambalda-style NiS exploration in Australia and these are imposed by the following factors (Trench and Williams, 1994):

a) the generally small size of the deposits (0.5 to $3.0 \mathrm{Mt}$ );

b) the extreme depth of weathering in the regolith; and

c) the abundance of anomalous responses from noneconomic targets.

We base the petrophysical characteristics of the Kambalda-style model on those electrical parameters described by Trench and Williams (1994). The surficial alluvial layer of the regolith is $12.5 \mathrm{~m}$ thick and has a conductivity of $0.05 \mathrm{~S} / \mathrm{m}$. This overlies a weathered basalt layer of $40 \mathrm{~m}$ thickness and a conductivity of $0.01 \mathrm{~S} / \mathrm{m}$. This regolith overlies a resistive basalt host rock of $0.001 \mathrm{~S} / \mathrm{m}$. Differential weathering of the ultramafic host of the NiS mineralization in the regolith is extended at depth into the basalt host rock. This weathered ultramafic has a conductivity of $0.05 \mathrm{~S} / \mathrm{m}$. The NiS ore, with a con- 


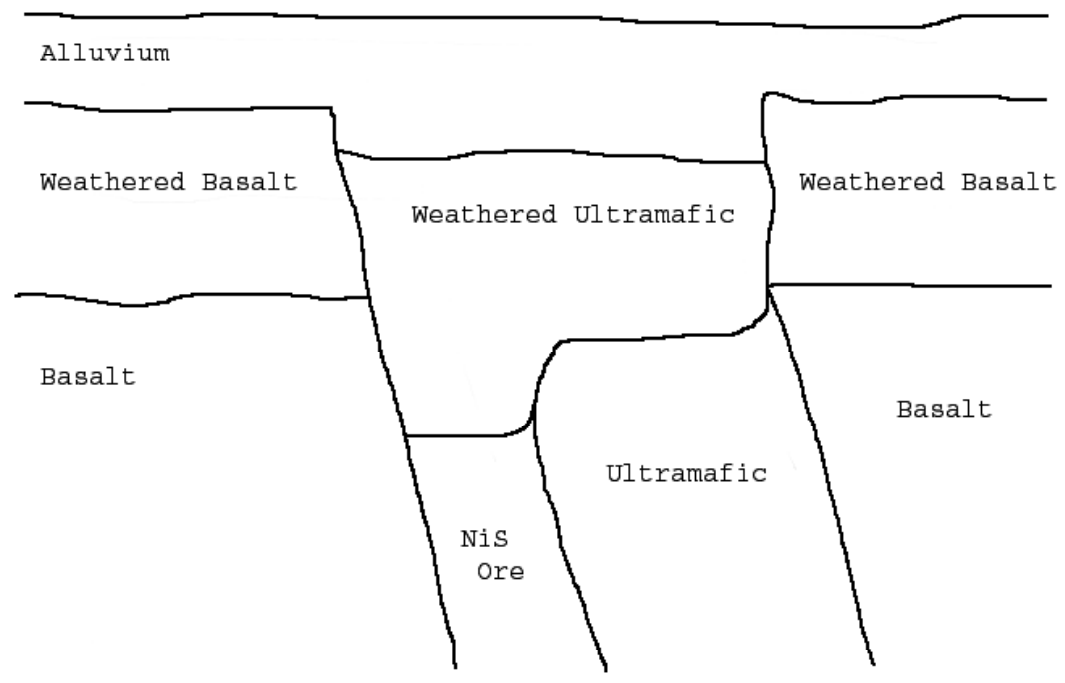

Figure 4-26 Cartoon of the cross-section of the Kambalda NiS deposit after Trench and Williams (1994).

ductivity of $1 \mathrm{~S} / \mathrm{m}$, and an unweathered ultramafic mineralization host with a conductivity of $0.01 \mathrm{~S} / \mathrm{m}$, extend at depth and with steep dip beneath the weathered ultramafic host. The NiS ore and ultramafic host have a $700 \mathrm{~m}$ strike length in the $x$ direction. Figure 4-26 is a cartoon of the cross-section of the Kambalda deposit from Trench and Williams (1994). Figure 4-27 presents a resistivity cross-section of the Kambalda-style model studied in this paper along the profile $y=0 \mathrm{~m}$.

We have designed various models based on a single initial model of the Kambalda deposit, presented in Figure 4-27, to test the usability of the software and to catalog the modeling results. Four separate forward modeling runs using INTEM3DIP were executed based on varying parameters of the ore body and background resistivities. All models show a horizontal 3-layered background with varying thicknesses but fixed resistivities and IP phases of $[20,100,1000]$ Ohm-m and [5, 10, 5] mrad respectively. The overlying weathered ultramafic and adjacent ultramafic bodies were each given resistivities and IP phases of $[20,100]$ Ohm-m and $[5,20]$ mrad. Similar EM properties of the ore body are [10] Ohm-m and [100] mrad.

The forward modeling results are presented as apparent resistivity/phase pseudosections below. 


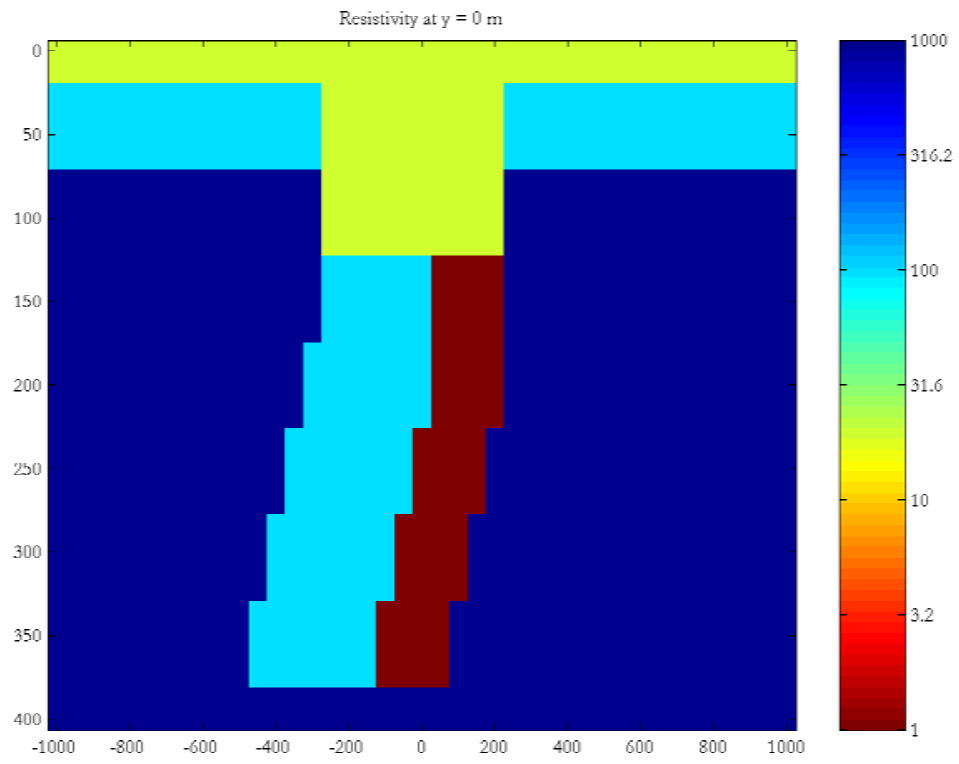

Figure 4-27 Cross-section of the total resistivity along the $y=0 \mathrm{~m}$ profile for the Kambalda-style NiS orebody used for forward modeling the FEM response.

\subsubsection{Trench-Williams Model 1 (TWM1)}

The initial model TWM1 was created from Figure 4-27, where the slanting of the ore body was represented by hexagons shifted with increasing depth. The vertical and longitudinal dimensions of the ore body were $250 \mathrm{~m}$ and $600 \mathrm{~m}$ respectively with a thickness of $200 \mathrm{~m}$. Model TWM1 is the basis model for the modeling surveys. The latter models show increased ore deposit geometries to analyze output effects.

A 3-D view of this model is shown in Figure 4-28, while Figure 4-29 presents the pseudosections of apparent resistivity and phase for this model. These pseudo sections provide a very complex image of the deposit, which is difficult to interpret without using an appropriate inversion method. Nevertheless, we can clearly see both the anomalous resistivity and IP effects in these sections.

\subsubsection{Trench-Williams Model 2 (TWM2)}

Model TWM2 is a manipulated version of TWM1 where the dx, dy, dz discretization has been increased to $50 \mathrm{~m}$ instead of $25 \mathrm{~m}$ and the layer thicknesses have been increased (Figure 4-30). These changes were made to facilitate computation "run time" and to slightly increase the depth of the ore body. The vertical and longitudinal dimensions of the ore body were $250 \mathrm{~m}$ and $600 \mathrm{~m}$ 


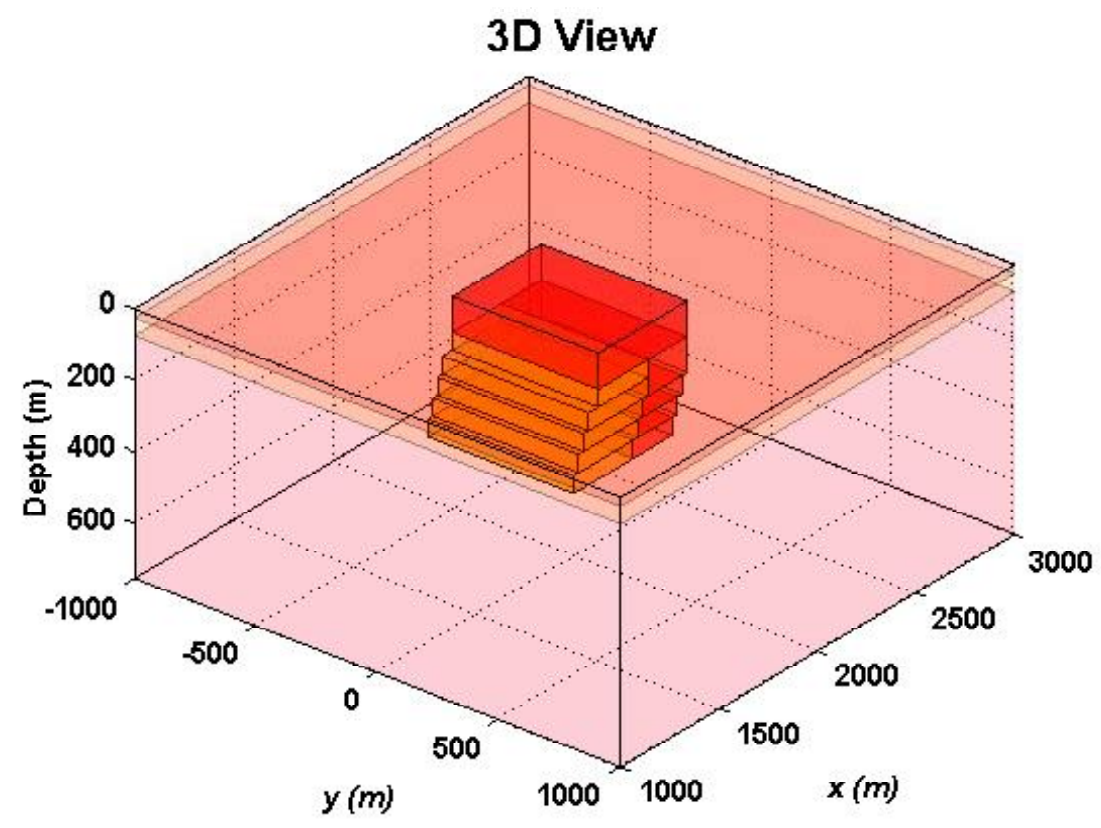

Figure 4-28 A 3-D view of Trench-Williams Model 1 (TWM1).

respectively with a thickness of $250 \mathrm{~m}$.

The pseudosections (Figure 4-31) are very similar to those for model TWM1, but with softer contrasts from the edge effects, particularly in the apparent resistivity plot.

\subsubsection{Trench-Williams Model 3 (TWM3)}

Model TWM3 has extended the ore deposit length from TWM2 by $100 \mathrm{~m}$ in both positive and negative y-directions (Figure 4-32). The upper two background layer thicknesses have also been doubled to $50 \mathrm{~m}$ and $100 \mathrm{~m}$. The vertical and longitudinal dimensions of the ore body were $250 \mathrm{~m}$ and $800 \mathrm{~m}$ respectively with a thickness of $250 \mathrm{~m}$.

As the model size has been increased, it has become easier to facilitate computation as well as $3 \mathrm{D}$ visualization.

The pseudosection plots are reappearing in similar patterns, though, with introduced heterogeneities, particularly in the apparent resistivity plots (Figure 4-33). Note also that the edge effects are decreasing with larger geometries. 

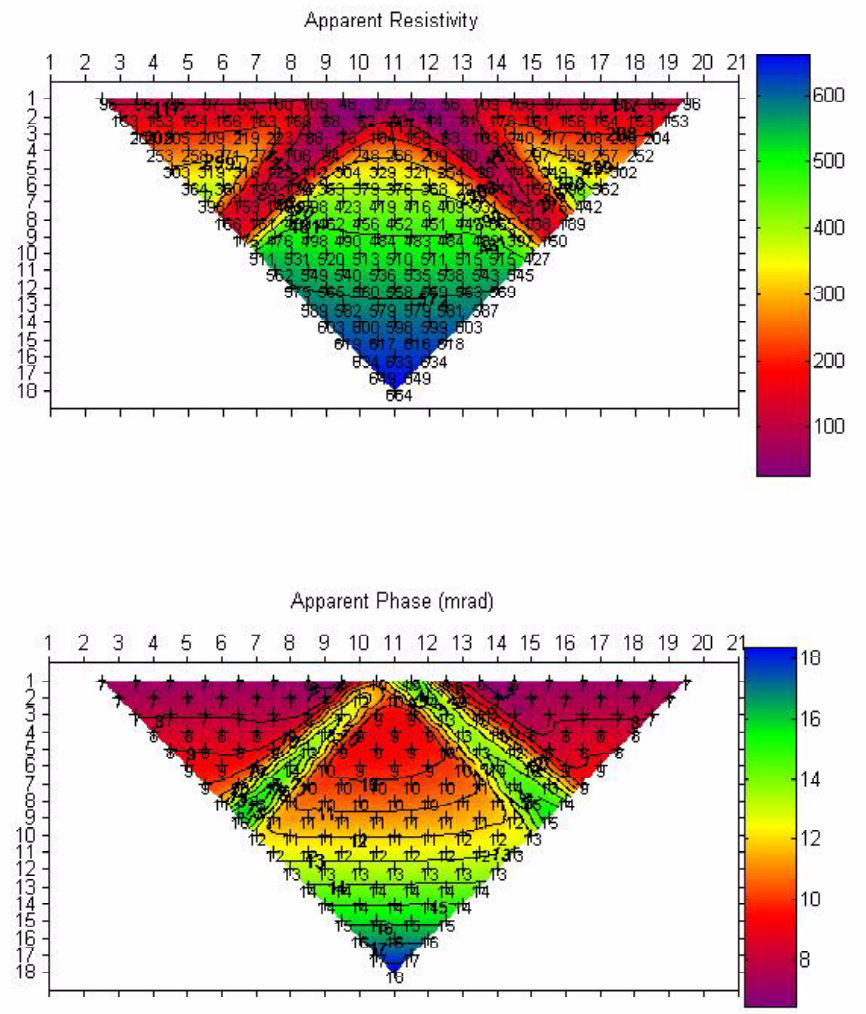

Figure 4-29 Pseudosections of apparent resistivity and phase for Trench-Williams Model 1. 


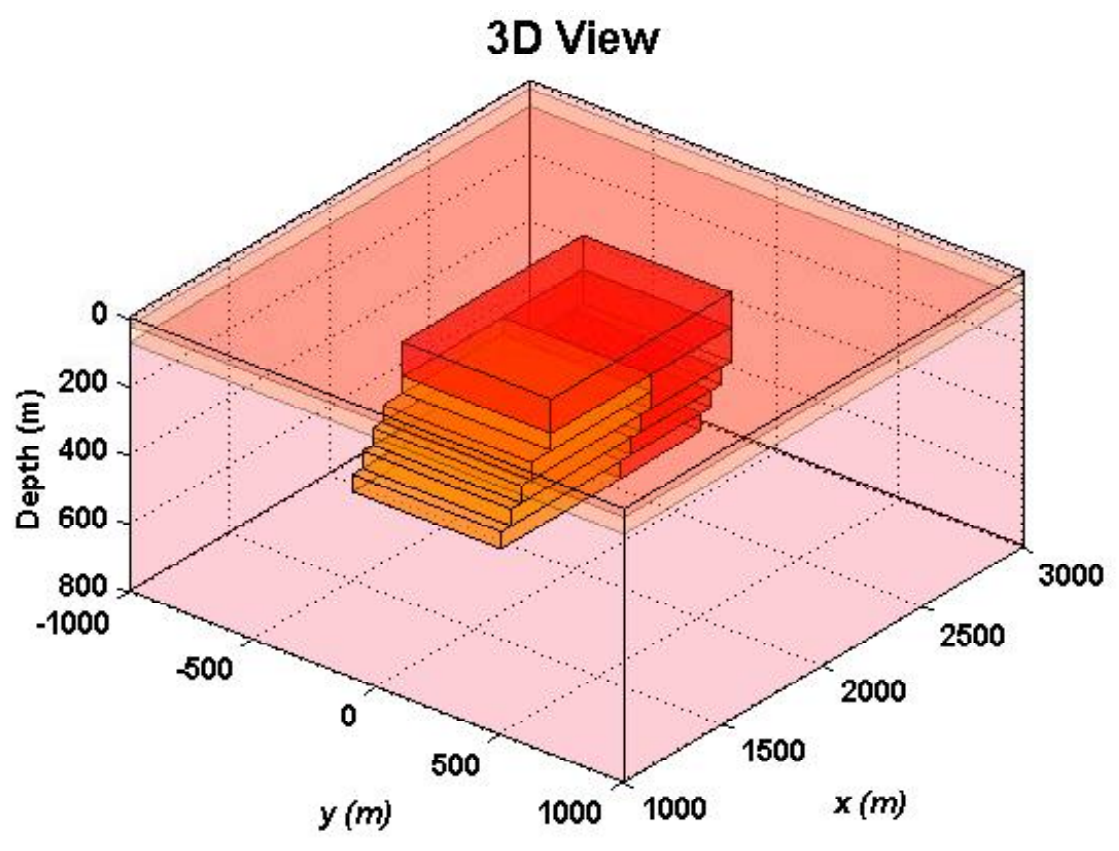

Figure 4-30 A 3-D view of Trench-Williams Model 2 (TWM2).

\subsubsection{Trench-Williams Model 4 (TWM4)}

Finally, model TWM4 was created with an interest in analyzing effects from an over-exaggerated model in depth (Figure 4-34). Though this model does not hold with the geometric parameters as set for in the original model, it was created with the intent of comparing these results with those of the smaller, more correct, models. The vertical and longitudinal thicknesses of the ore body were $500 \mathrm{~m}$ and $1000 \mathrm{~m}$ respectively with a thickness of $250 \mathrm{~m}$.

The depth of the lowermost layer in the ore deposit is at $650 \mathrm{~m}$. Computational "run time" was greatest for this particular model.

Figure 4-35 presents the corresponding pseudosection plots, computed for model TWM4. They show similar results as before, only here, the larger increase in apparent phase (as shown in blue) provided indication that the geometry of the ore deposit was resistively favored to one side. Model TWM1 showed this same phenomenon, but with a smaller phase contrast. 

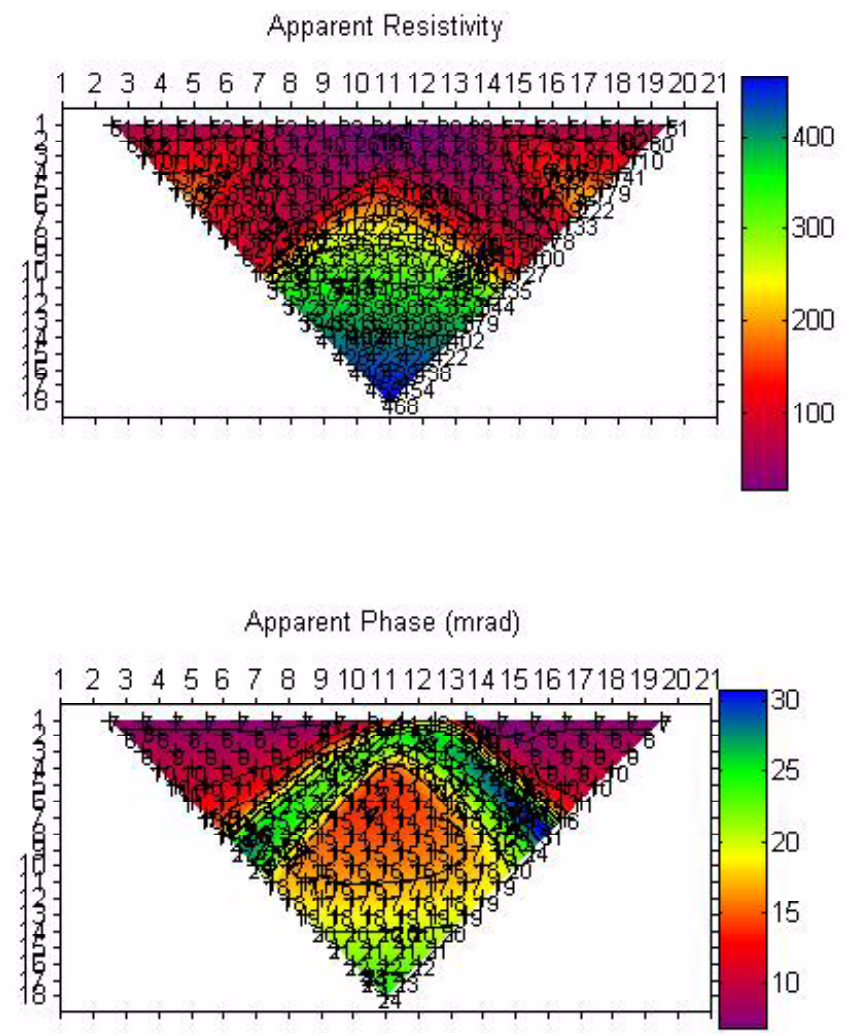

Figure 4-31 Pseudosections of apparent resistivity and phase for Trench-Williams Model 2. 


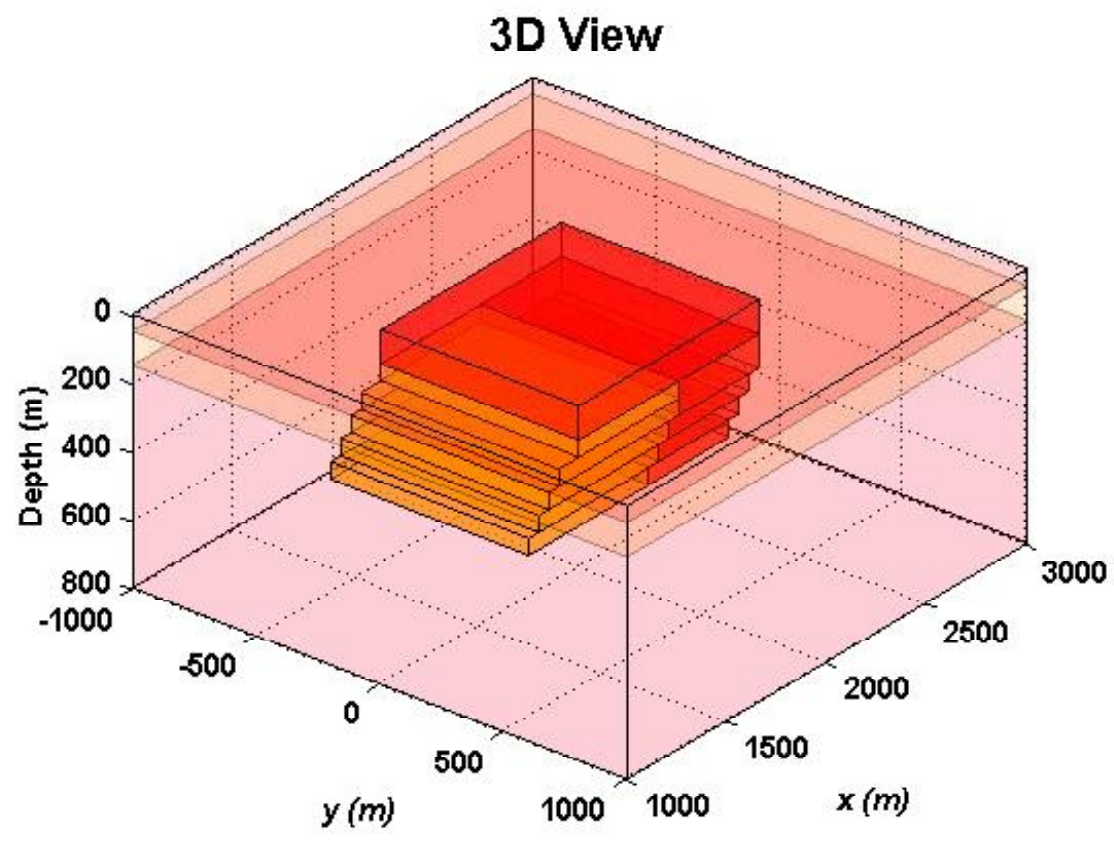

Figure 4-32 A 3-D view of Trench-Williams Model 3 (TWM3).
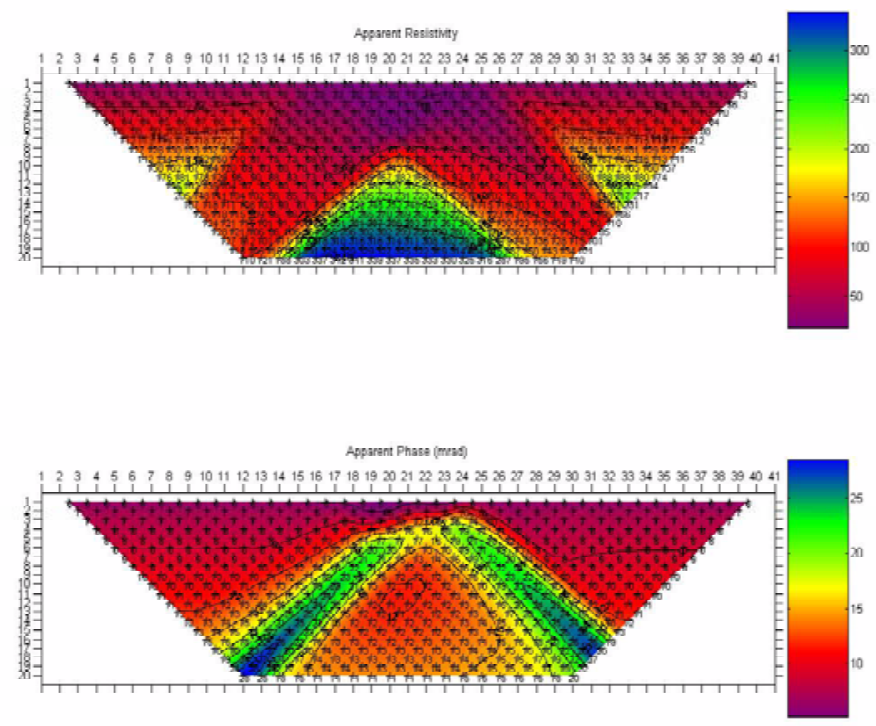

Figure 4-33 Pseudosections of apparent resistivity and phase for Trench-Williams Model 3. 


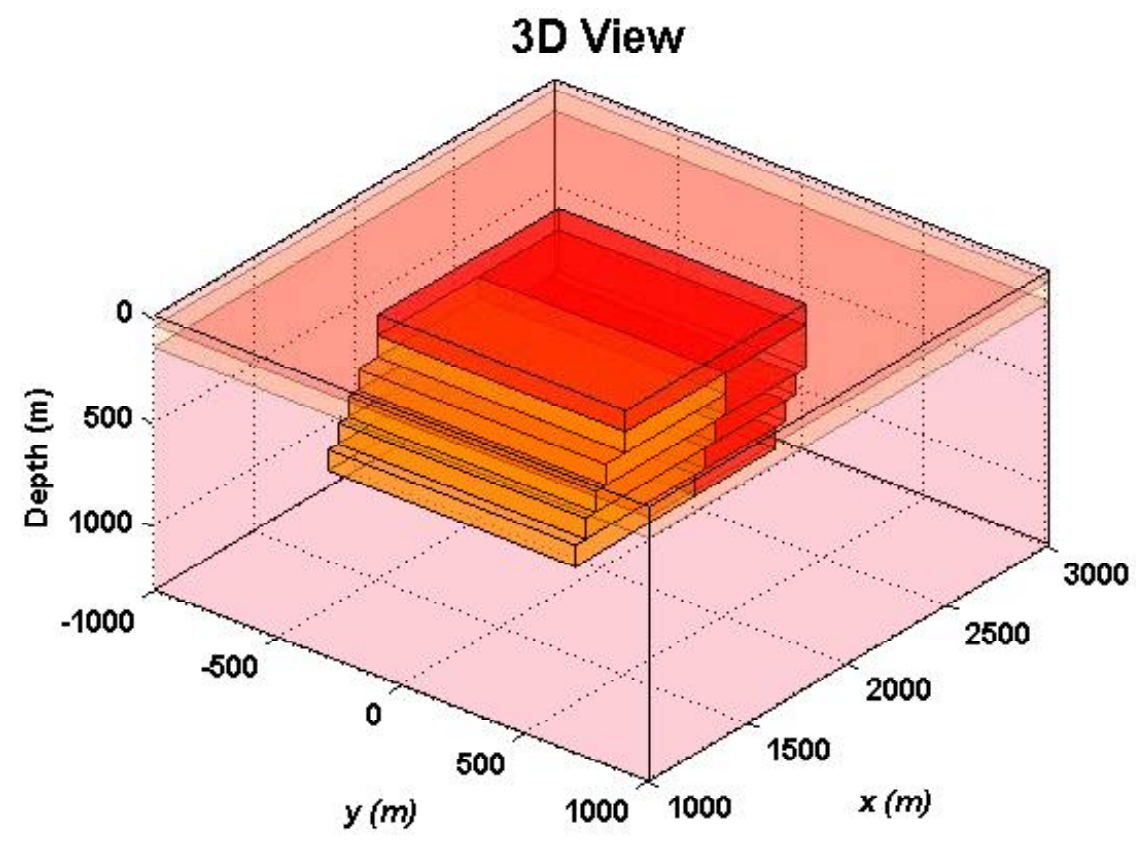

Figure 4-34 A 3-D view of Trench-Williams Model 4 (TWM4).
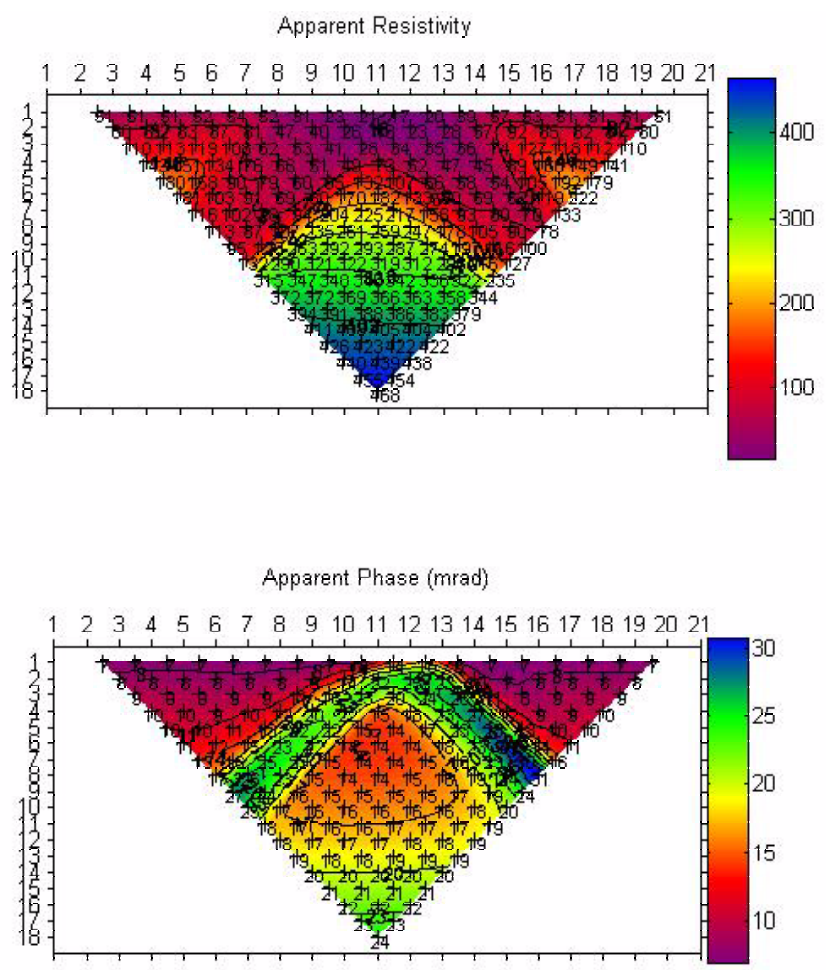

Figure 4-35 Pseudosections of apparent resistivity and phase for Trench-Williams Model 4. 


\section{RESULTS AND DISCUSSION}

During the first budget year of the project we have completed the work on PHASE I, task 1: construction and analysis of the reliable physical and mathematical models of the IP effect, based on the effective-medium theory. We have developed a rigorous mathematical model of multi-phase conductive media, which can provide a quantitative tool for evaluation of the type of mineralization, using the conductivity relaxation model parameters. The geoelectrical parameters of this model are determined by the intrinsic physical and geometrical characteristics of the composite medium: the conductivity contrast between the different phases of the medium, the size of the inclusions, and the closest distance between their centers. Therefore, in principle, the effective complex conductivity of this new model may serve as a basis for determining the intrinsic characteristic of the polarizable rock formation from the observed electrical data. As a result, the parameters of the new conductivity relaxation model can be used for discrimination of the different types of rock formations, which is an important goal in mineral exploration.

We have advanced the work on PHASE I, task 2: development of the 3-D EM-IP forward modeling system. We have developed a prototype 3-D EMIP modeling algorithm, based on the contraction integral equation method, which improves the convergence rate of the iterative solvers. This code can handle various types of sources and receivers to compute the effect of the complex resistivity model. We have tested the working version of this code, INTEM3DIP, for computer simulation of the IP data for several models of typical mineral deposits, including a resistivity/IP model of a typical porphyry copper system in the southwestern U. S., and a model of a Kambalda-style, nickel sulfide deposit.

The numerical modeling study helps us to build a foundation for future development of EM-IP modeling and inversion methods, directed at determining the electrical conductivity and the intrinsic chargeability distributions, as well as the other parameters of the relaxation model simultaneously. Future research will be focused on developing a parallelized version of this software which can be used for numerical simulation of the EM-IP effects for large-scale geoelectrical models typical for practical mineral exploration problems. 
RESULTS AND DISCUSSION 


\section{CONCLUSIONS}

The key accomplishments of the first research year of the project are as follows:

1. A new physical and mathematical model of electrical conductivity of the composite medium has been developed.

2. Asymptotic analysis of the composite medium conductivity has been conducted.

3. A working version of the 3-D EM-IP forward modeling system/code INTEM3DIP has been developed.

4. The new code, INTEM3DIP, is able to generate a theoretical IP response for complex 3-D geoelectrical models of the mining targets. The 3-D EM-IP responses have been computer simulated for two typical mineral deposits: a southwest U.S. porphyry deposit and a Kambalda-style nickel sulfide deposit.

It is essential for the success of this project to have a reliable conductivity relaxation model, quantitatively describing the relationships between the intrinsic characteristics of the heterogeneous rocks and their effective conductivity. The ability to model the EM-IP effect for complex 3-D geoelectrical structures with arbitrary conductivity distribution constitutes an important part of the quantitative interpretation of IP data in a complex 3-D environment. Thus, the results obtained so far provide a background for future successive development of new technology for mineral exploration and mineral discrimination, based on electromagnetic methods. 


\section{REFERENCES}

Cole, K. S., and Cole, R. H., 1941, Dispersion and absorption in dielectrics, J. Chem. Phys., 9, 343-351.

Hursán, G. and Zhdanov, M. S. , 2002, Contraction integral equation method in 3-D electromagnetic modeling: Radio Science, 37, 1089,

doi: $10.1029 / 2001 R S 002513$.

Kamenetsky, F. M., 1997, Transient geo-electromagnetics: GEOS, Moscow, $162 \mathrm{pp}$.

Luo Y., and Zang, G., 1998, Theory and application of spectral induced polarization: Society of Exploration Geophysicists, Tulsa, OK, 171 pp.

Marshall, D. J., and Madden, T. R., 1959, Induced polarization, a study of its causes: Geophysics, 24, 790-816.

McCracken, K. G., Oristaglio, M. L., and Hohmann, G. W., 1986, Minimization of noise in electromagnetic exploration systems: Geophysics, 51, 819-832.

Kolundzija, B. M., and Djordjevic, A. R., 2002, Electromagnetic modeling of composite metallic and dielectric structures: Artech House, Boston, London.

Nelson, P. H., 1997, Induced polarization research at Kennecott, 1965-1977: The Leading Edge, 16, 29-33.

Norris, A. N., Sheng, P., and Callegari, A. J., 1985, Effective-medium theories for two-phase dielectric media: J. Appl. Physics., 57, 1990-1996.

Pelton, W. H., 1977, Interpretation of induced polarization and resistivity data, Ph.D. thesis, University of Utah, 255 pp.

Pelton, W. H., Ward, S. H., Hallof, P. G., Sills, W. R., and P. H. Nelson, 1978, Mineral discrimination and removal of inductive coupling with mulifrequency IP: Geophysics, 43, 588-609.

Seigel, H. O., 1959, Mathematical formulation and type curves for induced polarization: Geophysics, 24, 547-565.

Sheinman, S. M., 1969, Contemporary physical foundations of the electrical prospecting theory: Nedra, Moscow, 224 pp.

Shuev, R.T., and Johnson, M., 1973, On the phenomenology of electrical relaxation in rocks: Geophysics, 38, 37-48.

Stolz, N., Raiche, A., Sugeng, F., and Macnae, J., 1995, Is full 3-D inversion necessary for interpreting EM data?: Explor. Geophys., 26, 167-171.

Stone, W. E., and Masterman, E. E., 1998, Kambalda nickel deposits, in Berkman, D. A. and Mackenzie, D. H., Eds., Geology of Australian and Papua New Guinean mineral deposits: Aust. Inst. Mining Metallurgy, 347-355.

Trench, A., and Williams, P. K., 1994, Application of geophysics to nickel sulphide exploration in the Kambalda district, Western Australia, in Denith, M. C., Frankcombe, K. F., Ho, S. E., Shepherd, J. M., Groves, D. I., and 
Trench, A., Eds., Geophysical signatures of Western Australian mineral deposits: Aust. Soc. Explor. Geophys. Special Publication No. 7, 169-179.

Wait, J. R., 1959, The variable-frequency method, in Wait, J.R., Ed., Overvoltage research and geophysical applications: Pergamon, Oxford.

Wait, J. R., 1982, Geo-electromagnetism: Academic Press, New York, 268 pp.

Zhdanov, M. S., Dmitriev, V. I., Fang, S., and Hursan, G., 2000, Quasianalytical approximations and series in electromagnetic modeling, Geophysics, 65, 1746-1757.

Zhdanov, M. S., 2002, Geophysical inverse theory and regularization problems: Elsevier, Amsterdam, New York, Tokyo, 628 pp.

Zhdanov, M. S., 2005, Development of new geophysical techniques for mineral explorations and mineral discrimination: SME Annual Meeting and Conference, Salt Lake City.

Zonge, K, and J. C. Wynn, 1975, Recent advances and applications in complex resistivity measurements: Geophysics, 40, 851-864. 